\title{
Quantum scattering and semiclassical transition state theory calculations on chemical reactions of polyatomic molecules in reduced dimensions
}

\author{
Samuel M. Greene, Xiao Shan, and David C. Clary
}

Physical and Theoretical Chemistry Laboratory, Department of Chemistry, University of Oxford, South Parks Road, Oxford, OX1 3QZ, United Kingdom

\section{Introduction}

In quantum reaction dynamics the Schrödinger equation is solved for the nuclei taking part in a chemical reaction for a given potential energy surface. The first such calculations were on the simplest reactions between atoms and diatomic molecules such as $\mathrm{H}+\mathrm{H}_{2}$ and $\mathrm{F}+\mathrm{H}_{2}$ for a collinear geometry [1-5]. These calculations illustrated quantum mechanical effects such as tunnelling and scattering resonances which were also found in later calculations in higher dimensions and with more atoms. The first converged quantum scattering calculations on a reaction in three dimensions was on $\mathrm{H}+\mathrm{H}_{2}$ [6] which has become a fundamental benchmark for theoretical and experimental studies on chemical reactions [7]. These calculations illustrated the power of quantum scattering calculations in being able to calculate essentially simultaneously differential cross sections selected in the quantum states of reactants and products as a function of collision energy and also kinetic quantities such as rate constants as a function of temperature. Subsequent calculations of this type on the $\mathrm{F}+\mathrm{H}_{2}$ reaction also highlighted exceptional agreement between theory and experiment even at the differential cross section level $[8,9]$. For 
atom+diatomic reactions such as these highly accurate potential energy surfaces are available [10] and theory is truly competitive with experiment.

In moving to calculations on reactions on polyatomic molecules, the $\mathrm{OH}+$ $\mathrm{H}_{2}$ reaction became the subject of many quantum scattering calculations [11-15]. These computations illustrated strong vibrational mode selective effects in the $\mathrm{H}_{2} \mathrm{O}$ products of the reaction. The quantum scattering calculations were carried out by solving both the time-independent and time-dependent Schrödinger equation. Initially the available potential energy surfaces for this reaction were not very accurate but there have been improvements in accuracy in recent years $[16,17]$. Several quantum scattering calculations on reactions of other polyatomic reactions were also carried out usually with approximations applied for the vibrational modes treated explicitly and the geometry considered [18-21]. In recent years the $\mathrm{H}+$ $\mathrm{CH}_{4}$ reaction has become something of a benchmark and has also been the subject of calculations using the Multi Configurational Time Dependent Hartree Fock Method [22] and the Ring Polymer Molecular Dynamics approach [23].

In moving on to more complicated reactions of polyatomic molecules a major challenge is having accurate potential energy surfaces available. Since rate constants depend exponentially on the height of the barrier in a potential energy surface only the most accurate $a b$ initio quantum chemistry computations will suffice and a very large number of such calculations need to be carried out to produce a reliable and global potential energy surface in all degrees of freedom.

One powerful and more computationally inexpensive approach is to treat the quantum scattering calculations of polyatomic reactions in reduced dimensions (RD) and also obtain the potential energy surface, using accurate quantum chem- 
istry methods, expressed in the same reduced dimensions [24, 25]. This approach is justified through the quantum scattering calculations carried out on reactions such as $\mathrm{OH}+\mathrm{H}_{2}$ where it was found that it was not necessary to treat all degrees of freedom explicitly in quantum scattering calculations to calculate reliable experimentally important quantities such as rate constants $[11,26]$. This method is a general one and allows for quantum scattering calculations to be performed on a wide range of polyatomic reactions much more complicated than $\mathrm{H}+\mathrm{CH}_{4}$. In Section 2 of this article a description is given of some of the recent computations carried out with this reduced dimensionality approach.

Transition State Theory (TST) is a very powerful and general theory for calculating the rate constants of chemical reactions from a very minimal amount of information of the system at the transition state geometry [27]. There are also powerful extensions of the theory which can account for quantum mechanical tunnelling which nearly always is significant in reactions involving the abstraction or exchange of hydrogen atoms [28]. An alternative procedure for accounting for quantum mechanical tunnelling involving many degrees of freedom is Semiclassical Transition State Theory (SCTST) [29, 30]. This approach only requires the second, third and fourth derivatives of the electronic energy at the transition state and does not require as such a potential energy surface. The method was applied to a small number of reactions in the 1990s [31, 32] but the accuracy of the approach was not then very clear. However, there has been a recent resurgence of interest in this method mainly driven by the increasing availability and power of ab initio quantum chemistry methods $[33,34]$. By combining SCTST with RD procedures a particularly computationally inexpensive and powerful approach is 
obtained for calculating rate constants that retains the accuracy of multidimensional theory [35]. This approach is described in Section 3, where the accuracy of the semiclassical tunnelling procedure is also tested by comparison with the quantum scattering reduced dimensionality calculations on the hydrogen abstraction and exchange reactions of a variety of hydrocarbon molecules. Conclusions are given in Section 4.

\section{Quantum Scattering Calculations on Polyatomic Reactions in Reduced Dimensions}

\subsection{Methods}

\subsubsection{Scattering Theory and Coordinate Systems}

Within the RD framework, only a subset of internal degrees of freedom are treated explicitly in the quantum reactive scattering (QRS) calculation. Here we discuss the two-dimensional (2-D) case. In hyperspherical coordinates, the nuclear motion Hamiltonian is given by [36]:

$$
\hat{H}=-\frac{1}{2 \mu}\left(\frac{\partial^{2}}{\partial \rho^{2}}+\frac{1}{\rho^{2}} \frac{\partial^{2}}{\partial \delta^{2}}-\frac{3}{4 \rho^{2}}-\frac{\hat{J}^{2}}{\rho^{2}}\right)+V(\rho, \delta) .
$$

where $\rho$ and $\delta$ are the hyperspherical radius and angle respectively, and $\hat{J}$ is the total angular momentum operator, and $\mathrm{V}(\rho, \delta)$ is the potential energy. The discussions on constructing a 2-D potential energy surface (PES) will be given in the next section. The detailed explanations on how Eq. 1 is solved can be found elsewhere. Here we only give a brief outline of the $R$-matrix propagation scheme developed by Stechel, Walker and Light [36], the application of the approximate boundary conditions and the $J$-shifting model [37-40]. 
To perform $R$-matrix propagation, the hyperspherical coordinate space is firstly divided into sectors in $\rho$ with even width. The $\delta$-dependent Hamiltonian for each sector, $\rho_{i}$, is given by:

$$
\hat{H}_{\delta}=-\frac{1}{2 \mu \rho_{i}^{2}} \frac{\partial^{2}}{\partial \delta^{2}}+V\left(\rho_{i}, \delta\right) .
$$

In each sector, the wave function for quantum state $k$ is expanded as a function of $\rho$ :

$$
\Psi_{k}\left(\rho, \delta ; \rho_{i}\right)=\sum_{k^{\prime}}^{N} f_{k^{\prime} k}\left(\rho ; \rho_{i}\right) \phi_{k^{\prime}}\left(\delta ; \rho_{i}\right) .
$$

The $\delta$-dependent wave function, $\phi_{k^{\prime}}\left(\delta ; \rho_{i}\right)$, is obtained by diagonalising Eq. 3 using a discrete variable representation with a particle-in-a-box basis [41-43]. The size of the contracted basis, $N$, is chosen to be large enough so that at a given energy all open channels of the system are propagated [36]. Combining Eqs. 2 and 3, the problem is now one of solving the close-coupled equations:

$$
\frac{d^{2}}{d \rho^{2}} \mathbf{f}\left(\rho, \rho_{i}\right)+\mathbf{W}\left(\rho_{i}\right) \mathbf{f}\left(\rho, \rho_{i}\right)=0
$$

where $\mathbf{W}$ is a diagonal matrix that depends on the initial total energy $E$ and the hyperspherical adiabats in sector $i$. It is given by:

$$
W_{k^{\prime} k}\left(\rho_{i}\right)=2 \mu\left(E-\epsilon_{k}\left(\rho_{i}\right)-\frac{3}{8 \mu \rho_{i}^{2}}-\frac{J(J+1)}{2 \mu \rho_{i}^{2}}\right) \delta_{k k^{\prime}}
$$

To solve the close-coupled equations, the $R$-matrix is propagated through all sectors from the interaction region to the asymptotic region $[36,44]$.

At large enough value of $\rho$, in principle, the $R$-matrix should be projected into Jacobi coordinates whereupon the boundary conditions may be imposed. However, this projection can be complicated [45], the relationship between the Jacobi and hyperspherical coordinates is discussed later in this section. It has been found that 
applying the boundary conditions directly in the hyperspherical coordinates can be a good approximation $[11,46]$. The scattering matrix is then defined as:

$$
\mathbf{S}\left(\rho_{i}\right)=\left(\mathbf{R}\left(\rho_{i}\right) \mathbf{O}^{\prime}\left(\rho_{i}\right)-\mathbf{O}\left(\rho_{i}\right)\right)^{-1}\left(\mathbf{R}\left(\rho_{i}\right) \mathbf{I}^{\prime}\left(\rho_{i}\right)-\mathbf{I}\left(\rho_{i}\right)\right)
$$

The asymptotic incoming and outgoing wave functions are given by:

$$
\begin{gathered}
I_{k k}\left(\rho_{i}\right)=\lambda_{k}^{-1 / 2} \exp \left(-\mathrm{i} \lambda_{k} \rho_{i}\right), \\
O_{k k}\left(\rho_{i}\right)=\lambda_{k}^{-1 / 2} \exp \left(\mathrm{i} \lambda_{k} \rho_{i}\right) .
\end{gathered}
$$

where $\lambda_{k}\left(\rho_{i}\right)=\sqrt{W_{k k}\left(\rho_{i}\right)}$. The reaction probability matrix, $P_{i \rightarrow f}^{J}\left(E, \rho_{i}\right)$ is defined as:

$$
P_{i \rightarrow f}^{J}\left(E ; \rho_{i}\right)=\left|S_{i \rightarrow f}^{J}\left(E ; \rho_{i}\right)\right|^{2}
$$

The subscripts ' $i$ ' and ' $f$ ' denote respectively the initial reactant and final product states. It should be noted that since approximate boundary conditions are applied directly in the hyperspherical coordinates, oscillatory behaviour in $P_{i \rightarrow f}^{J}$ can be observed as a function of $\rho_{i}$ [47]. It has been shown previously that such an effect can be minimized by averaging $S_{i \rightarrow f}^{J}$ over a number of sectors in the asymptotic region $[48,49]$.

Once $S_{i \rightarrow f}^{J}$ or $P_{i \rightarrow f}^{J}$ is obtained from the $R$-matrix propagation scheme, the state-to-state reaction integral cross sections can be easily calculated using:

$$
\sigma_{i \rightarrow f}\left(E_{C}^{i}\right)=\frac{\pi}{q_{i}^{2}} \sum_{J=0}^{J_{\max }}(2 J+1) P_{i \rightarrow f}^{J}(E),
$$

where $E_{C}^{i}\left(=E-\varepsilon_{i}\right)$ is the collision energy, and $q_{i}^{2}=2 \mu_{R}\left(E-\varepsilon_{i}\right)$.

In practice, to ensure convergence with respect to the total angular momentum, $J$, the value of $J_{\max }$ is usually over 200 for multi-atomic systems. Since the $R$ matrix propagation scheme requires iteration over the $\rho$-sectors as well as the 
$J$-values, the computation can be expensive for large reactions. When only the reaction rate constant is of interest, the computation can be simplified via the $J$-shifting model $[50,51]$, where only the properties at $J=0$ are calculated, and the cumulative reaction probability is given by:

$$
P_{\text {cum }}^{J=0}(E)=\sum_{i, f}\left|S_{i \rightarrow f}^{J=0}(E)\right|^{2} .
$$

The QRS rate constant is then calculated using this cumulative reaction probability as follows $[50,51]$ :

$$
k_{\mathrm{QRS}}(T)=\frac{Q_{\mathrm{tot}}^{\ddagger(m)}}{h Q_{\mathrm{tot}}^{\text {react }}} \int_{0}^{\infty} P_{\text {cum }}^{J=0}(E) \exp \left(-\frac{E}{k_{\mathrm{B}} T}\right) \mathrm{d} E,
$$

where $Q_{\text {tot }}^{\text {react }}$ is the total partition functions of the reactants, while $Q_{\text {tot }}^{\ddagger(m)}$ is the partition function of the TS. It should be noted that only $m=3 N-6-2$ modes are treated in the vibrational partition function of the TS. The other two modes, one of which corresponds to motion along the reaction path, are treated explicitly in the QRS calculation and their contribution to the rate constants are included in the cumulative reaction probability, $P_{\text {cum }}^{J=0}(E)$.

The classical transition state theory (TST) rate constant is often calculated and compared to the QRS rate constant to analyse the contribution from quantum effects. It is given by:

$$
k_{\mathrm{TST}}(T)=\frac{Q_{\text {tot }}^{\ddagger\left(m^{\prime}\right)}}{h Q_{\mathrm{tot}}^{\text {react }}} \times k_{\mathrm{B}} T \times \exp \left(-\frac{\Delta V_{a}^{\ddagger}}{k_{\mathrm{B}} T}\right) .
$$

where $\Delta V_{a}^{\ddagger}$ is the adiabatic vibrational barrier height. The TS vibrational partition function, all the vibrational modes except the one corresponding to the reaction paths are included in the TS vibration partition function, and $m^{\prime}=3 N-6-1$. 


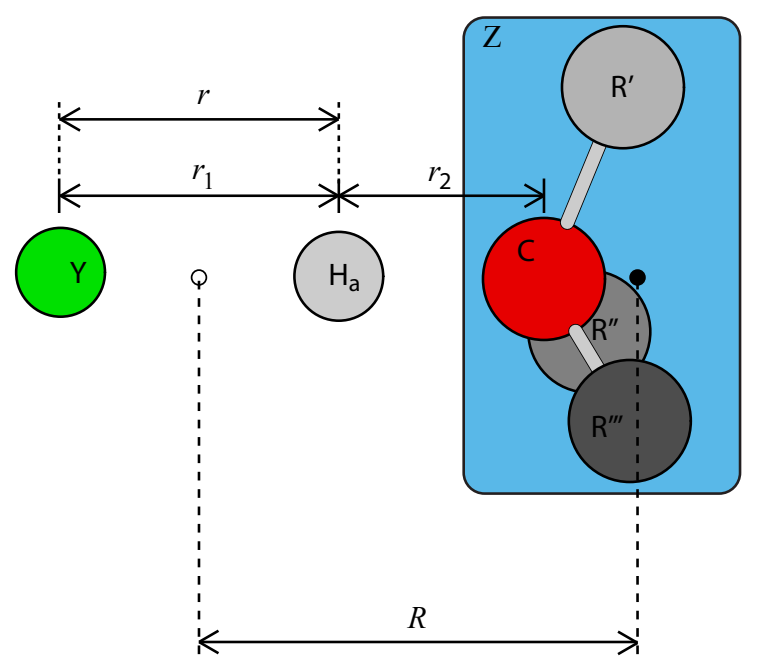

Figure 1: Schematic representation of the H-abstraction or H-exchange reaction.

Note that the vibrational frequencies in the calculation of TS partition functions in equations (14) and (15) are slightly different, because a projection scheme is applied in the construction of the PES, which in turn is used the QRS calculation. This scheme and the PES construction will be discussed in the next section. We now turn our attention to the coordinate systems that are used in the calculation.

Figure 1 shows a generalised representation of a H-abstraction or H-exchange reaction. The changes in the two bond lengths, $r_{1}$ and $r_{2}$, are the two internal DOFs that are treated explicitly in our RD QRS calculations. They correspond to the chemical bonds formed and broken during the reaction. The Jacobi coordinates are also shown in Figure 1 as $r$ and $R$. In principle $r$ and $R$ correspond to the distance between the $\mathrm{H}$-atom and the centre of mass (CoM) of the fragment $\mathrm{Y}$ and between CoMs of $\mathrm{Y}$ and $\mathrm{Z}$. Note that for $\mathrm{H}$-abstraction reactions $r$ is simply $r_{1}$. In practice, for convenience $R$ can also be defined relative to the $\mathrm{C}$-atom in $\mathrm{Z}$ to which the leaving H-atom is attached [25, 52-55]. The Jacobi coordinates can 
then be converted into hyperspherical coordinates using:

$$
\frac{m_{1}}{\mu} R^{2}=(\rho \cos \delta)^{2}, \frac{m_{2}}{\mu} r^{2}=(\rho \sin \delta)^{2}
$$

with

$$
m_{1}=\frac{m_{\mathrm{YH}} m_{\mathrm{Z}}}{m_{\text {tot }}}, m_{2}=\frac{m_{\mathrm{H}} m_{\mathrm{Y}}}{m_{\mathrm{HY}}}, m_{3}=\frac{m_{\mathrm{Y}} m_{\mathrm{Z}}}{m_{\mathrm{Y}}+m_{\mathrm{Z}}}, \mu=\sqrt[3]{m_{1} m_{2} m_{3}} .
$$

\subsubsection{Potential Energy Surfaces and Computational Details}

In the previous section, we focused on solving the nuclear motion Schrödinger equation (i.e. the QRS calculation). The accuracy of such QRS calculations is largely dependent on the PES, i.e. the term $V(\rho, \delta)$ in Eq. 1. Despite the recent efforts and advances in the construction of multidimensional PES [56-59], it remains one of the biggest challenges in chemical dynamic and kinetic studies. One of the advantages in a RD calculation is that only a subset of (usually two) internal degrees of freedom, usually called the active modes, are explicitly treated. The number of grid points required for constructing the PES is therefore greatly reduced [50, 60-64]. This allows kinetic studies of chemical reactions that involve several atoms, for instance 15 atoms for the $\mathrm{H}+\mathrm{C}_{4} \mathrm{H}_{8}$ reaction [65]. The contribution of the remaining internal degrees of freedom, usually called the spectator modes, is included in the PES as their total zero-point energies (ZPE). Two types of PES construction schemes has been developed and tested over the years.

We first give a brief outline of the traditional or common procedure of producing $V(\rho, \delta)$ that involves fitting a parameterised function to ab initio data $[25,37-$ $39,47,55,66,67]$ 
- A set of grid points in hyperspherical coordinates are chosen. The coordinates of each grid point are then converted into bond lengths, $r_{1}$ and $r_{2}$, using Eqs. (14) and (15). Partial geometry optimization is then performed with the value of $r_{1}$ and $r_{2}$, i.e. the active modes, frozen.

- For each optimized geometry, a Hessian calculation is performed at the same level of theory as the geometry optimization. The contribution of the active modes to the spectator modes is projected out using either the rectilinear or curvilinear projection scheme. The post-projection spectator mode ZPE is then calculated.

- A single point energy calculation at higher level of theory is performed at each optimized geometry. The result of this calculation is added to the spectator mode ZPE to give the ab initio energy at each grid point.

- A parameterised potential function, for instance a 2-D double Morse potential, is fitted to the ab initio data. This fitted function is then used as $V(\rho, \delta)$ in the QRS calculation.

This approach of PES construction in general requires 60 to 150 grid points for a 2-D surface depending on the complexity of the system. For large systems that consists of more than 10 atoms, the $a b$ initio calculations may become computationally expensive. In addition, fitting a 2-D double Morse function with more than 25 parameters is by no means an easy task. Whether or not a global minimum is obtained in such a fitting is largely dependent on the initial guesses of the parameter values. However, it should also be noted that this approach, with 
carefully chosen grid points, normally contains information of the PES in the high energy region, and hence can potentially provide important insights into the energy landscape of a reaction.

To overcome the difficulties in the fitting procedure outlined above, alternative methods were developed $[40,65,68]$. The so-called $(1+1) \mathrm{D}$ PES is constructed based on the energy profile of the minimum energy path (MEP), and the rest of the 2-D surface was approximated using either a harmonic function [40] or a Morse function $[65,68]$. This approach has some similarities to other methods, such as the Reaction Path Hamiltonian [69] and Reaction Surface Hamiltonian [70] methods. In terms of $a b$ initio calculations, one only needs to perform the frequency and high level single point energy calculations for the points along the MEP. Typically only 40 points are required. This method can significantly reduce the computational expense of $a b$ initio calculations, especially for large systems. However, the description of the PES in the high energy region is normally inaccurate, although the contribution to a chemical reaction from this region is normally negligible. A potential drawback of this method is that it requires a rather smooth MEP in hyperspherical coordinates [55]. It should be noted that although this approach provides an efficient way of constructing an RD PES that is able to yield accurate thermal rate constants, for analyses of detailed reaction dynamics such as reaction probabilities or cross sections the 2-D fitting model is much more reliable [40].

The ab initio calculations can be performed using any quantum chemistry packages, such as MOLPRO [71], Gaussian [72], CFOUR [73], etc. The geometry optimization and frequency calculations are usually carried out at the second order Møller-Plesset perturbation (MP2) level of theory with a cc-pVTZ correlation 
consistent basis set [74]. Single point energy calculations are carried out at either coupled cluster level including single, double and perturbative triple excitations $[\mathrm{CCSD}(\mathrm{T})]$ with normal and augmented cc-pVTZ basis sets or explicitly correlated coupled cluster method, such as the CCSD(T)-F12a/cc-pVTZ-F12 method [75, 76], where cc-pVTZ-F12 stands for the triple- $\zeta$ correlation consistent F12 MOLPRO basis set of Peterson et al [77]. Note that other levels of theory as well as different basis sets can be employed for different reactions. For instance $d$-polarization functions can be added in the calculation of Cl-atom [66, 67].

\subsection{Results and Discussion}

\subsubsection{Application of the $(1+1)$ D PES}

In Section 2.1.2, we discussed the latest improvement in PES construction, the $(1+1) D$ method. von Horsten et al. [40] first developed the method and applied it to the $\mathrm{H}$ abstraction reactions by $\mathrm{H}$-atom from methane, ethane and propane molecules. Very good agreement was found between the $(1+1) \mathrm{D}$ QRS and the experimental rate constants for all the reactions. The use of a Morse function in the $(1+1) \mathrm{D}$ calculation were later proposed by Shan et al. [65] in the study of the $\mathrm{H}+\mathrm{n}-\mathrm{C}_{4} \mathrm{H}_{10} \longrightarrow \mathrm{H}_{2}+1-\mathrm{C}_{4} \mathrm{H}_{9} / 2-\mathrm{C}_{4} \mathrm{H}_{9}$ reactions. The same method was then

applied to the $\mathrm{H}_{2}+\mathrm{CF}_{3} \longrightarrow \mathrm{H}+\mathrm{HCF}_{3}$ reaction [68]. In Figure 2(a) we compare the QRS rate constants to the TST results. It can be seen that the two converge at high temperature, $T$, at which quantum effects have small contributions to the reaction. As $T$ decreases, typically below $500 \mathrm{~K}$, quantum effects becomes significant and the QRS rate constants are clearly higher than the TST ones. We also show the comparison between our calculated thermal rate constants and the 
experimental [78-80] and other theoretical [81] results. The experimental results from both Hranisavljevic et al. [78] and Hidaka et al. [79] were both done at $T$ above $1000 \mathrm{~K}$, and they have deviation as large as $\Delta \log k \sim 1.2$ between their results. Our results are in reasonable agreement with them. Lower $T$ experimental rate constants were reported by Arthur et al. [80] are shown in Figure 2(a) as the dotted-dashed blue curve. Our QRS results are in very good agreement with this set of data. Maity et al. [81] performed a canonical variational transition state theory calculation with small curvature tunneling approximations on this reaction, shown as the dotted green curve. However they predicted a much lower barrier height and the overestimation of the rate constants is clear at $T$ below $500 \mathrm{~K}$. The kinetic isotope effect was also studied for this reaction, and the rate constant ratio, $k_{\mathrm{H}_{2}} / k_{\mathrm{D}_{2}}$ is shown in Figure 2. Here $k_{\mathrm{H}_{2}}$ and $k_{\mathrm{D}_{2}}$ are the rate constants for the $\mathrm{H}_{2}+$ $\mathrm{CF}_{3} \longrightarrow \mathrm{H}+\mathrm{HCF}_{3}$ and $\mathrm{D}_{2}+\mathrm{CF}_{3} \longrightarrow \mathrm{D}+\mathrm{DCF}_{3}$ reactions, respectively. We compare our results to the experimental data reported by Kibby et al. [82]. The QRS rate ratio is in much closer agreement to the experimental data than the TST rate ratio. As expected, quantum effects contribute more to the rate of the H-atom abstraction reaction than its D-atom counterpart, and such effects are accurately captured in our QRS calculation.

\subsubsection{Application of the 2D fitted PES}

Despite the success of the $(1+1) \mathrm{D}$ method demonstrated in the last section, the fitting of an analytical potential function to a set of $a b$ initio data remains the common method for constructing a 2D PES. In the earlier RD studies, a lot of the efforts have been devoted to developing potential energy functions that can 

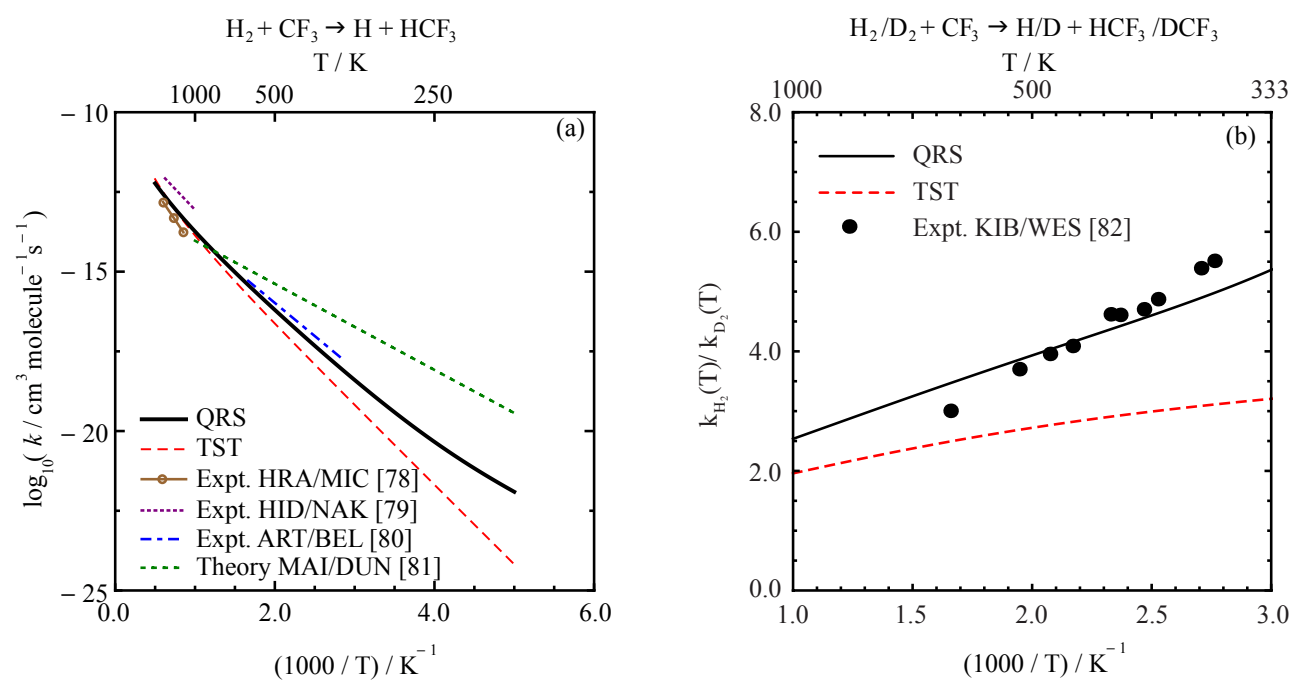

Figure 2: Comparison of (a) the calculated quantum thermal rate constants with the literature values the $\mathrm{H}_{2}+\mathrm{CF}_{3} \longrightarrow \mathrm{H}+\mathrm{HCF}_{3}$ reaction at $500 \mathrm{~K}$ to $2000 \mathrm{~K}$ and (b) the rate constant ratio with experimental results at $333 \mathrm{~K}$ to $1000 \mathrm{~K}$.

more accurately fit the $a b$ initio data $[39,52,83]$. In the past decade, our group has been using two types of 29-parameter double Morse functions [37-39, 47, 55, $66,67]$ that only differ by the definition at the position of the TS. These types of potential energy functions can often provide a more realistic representation of the PES in both the reactant and product channels by including any van der Waals (vdW) complexes that may exist. Using these fitting functions, we applied the RD QRS calculation to various chemical reactions that involve $\mathrm{H}$-atom abstraction or exchange. The reactions studied include $\mathrm{H}+\mathrm{CH}_{4} \longrightarrow \mathrm{H}_{2}+\mathrm{CH}_{3}$ [37], $\mathrm{Cl}+$ $\mathrm{CH}_{4} / \mathrm{CHD}_{3} \longleftrightarrow \mathrm{HCl}+\mathrm{CH}_{3} / \mathrm{CD}_{3}[47,66,67], \mathrm{CH}_{3}+\mathrm{CH}_{4} \longrightarrow \mathrm{CH}_{4}+\mathrm{CH}_{3}$ $[38,39]$ and $\mathrm{H}+\mathrm{cyc}-\mathrm{C}_{3} \mathrm{H}_{6} \longrightarrow \mathrm{H}_{2}+$ cyc $-\mathrm{C}_{3} \mathrm{H}_{5}$ [55]. Here we discuss two recent applications of this type of PES construction scheme.

The $\mathrm{H}$ abstraction from $\mathrm{CH}_{4}$ by $\mathrm{Cl}$-atom is a major Cl-radical depletion mech- 
anism in the atmosphere, and has therefore been widely studied both theoretically $[47,66,67,84-90]$ and experimentally [67, 89, 91-95]. Studies have been mainly on the ground electronic state reaction. Recently, several studies analysed the role of nonadiabatic processes for this reaction involving the spin-orbit state $\mathrm{Cl}\left({ }^{2} \mathrm{P}_{1 / 2}\right)$. Remmert et al. [66] developed a multiple-surface quantum scattering method within the RD framework. This method has been successfully applied to both the $\mathrm{Cl}\left({ }^{2} \mathrm{P}_{\mathrm{J}}\right)+\mathrm{CH}_{4}$ [66] as well as the $\mathrm{Cl}\left({ }^{2} \mathrm{P}_{\mathrm{J}}\right)+\mathrm{HCD}_{3}$ reactions [67]. These reactions contain three, two-fold degenerate PESs due to the unpaired electron in the valence $p$ orbital of the Cl-atom. To accommodate this multi-surface feature, the potential energy term, $V(\rho, \delta)$, in Eq. (2) now becomes a potential matrix:

$$
\mathbf{V}(\rho, \delta)=\left(\begin{array}{ccc}
V_{\mathrm{A} 1}^{\mathrm{d}} & \mathrm{A} & \mathrm{A} \\
\mathrm{A} & V_{\mathrm{E}}^{\mathrm{d}} & \mathrm{B} \\
V_{\mathrm{E}}^{\mathrm{d}} & \mathrm{B} & \mathrm{B}
\end{array}\right)
$$

Here $V_{\mathrm{A} 1}^{\mathrm{d}}$ and $V_{\mathrm{E}}^{\mathrm{d}}$ are the potential energy functions of the ground diabatic state and degenerate excited diabatic states respectively, and $\mathrm{A}$ and $\mathrm{B}$ are the spin-orbit coupling (SOC) potential terms between $\mathrm{A}_{1}$ and E states and between the E states respectively. The Schrödinger equation for a multi-surface system can be written in vector form:

$$
T\left(\begin{array}{l}
\Psi_{1 v} \\
\Psi_{2 v} \\
\Psi_{3 v}
\end{array}\right)+\left(\begin{array}{ccc}
V_{\mathrm{A} 1}^{\mathrm{d}} & \mathrm{A} & \mathrm{A} \\
\mathrm{A} & V_{\mathrm{E}}^{\mathrm{d}} & \mathrm{B} \\
V_{\mathrm{E}}^{\mathrm{d}} & \mathrm{B} & \mathrm{B}
\end{array}\right)=E\left(\begin{array}{l}
\Psi_{1 v} \\
\Psi_{2 v} \\
\Psi_{3 v}
\end{array}\right)
$$

In this particular calculation, the correlation consistent basis set cc-pV(T+d)Z$\mathrm{dk}$ is used for both geometry optimizations and frequency calculations. Here $(+\mathrm{d})$ indicates additional $d$-polarization functions for the Cl-atom [96] and (-dk) refers to the use of Douglas-Kroll scalar relativistic basis sets. Both cc-pV(T+d)Z-dk and cc-pV $(\mathrm{Q}+\mathrm{d}) \mathrm{Z}-\mathrm{dk}$ basis sets were used for the energy calculations. The results 
were extrapolated to achieve the basis set convergence via the method of Halkier et al. [97]. In the QRS calculation for the $\mathrm{Cl}+\mathrm{CHD}_{3} \longrightarrow \mathrm{HCl}+\mathrm{CD}_{3}$ reaction [67], a primitive basis of $N_{\delta}=200$ particle-in-a-box functions were used over $N_{\rho}=750$ sectors ranging from $\rho_{\min }=8 a . u$. to $\rho_{\max }=14 a . u$.. A total of 35 contracted basis were used and the maximum total angular momentum $J_{\max }$ of 300 was chosen to ensure the convergence of the calculation.

Figure 3(a) shows the integral cross sections (ICS) for the following three state-to-state reactions: $\mathrm{R} 1: \mathrm{Cl}\left({ }^{2} \mathrm{P}_{3 / 2}\right)+\mathrm{H}-\mathrm{CD}_{3}(v=0) \longrightarrow \mathrm{HCl}\left(v^{\prime}=0\right)+$ $\mathrm{CD}_{3} ; \mathrm{R} 2: \mathrm{Cl}\left({ }^{2} \mathrm{P}_{3 / 2}\right)+\mathrm{H}-\mathrm{CD}_{3}(v=0) \longrightarrow \mathrm{HCl}\left(v^{\prime}=1\right)+\mathrm{CD}_{3} ; \mathrm{R} 3: \mathrm{Cl}\left({ }^{2} \mathrm{P}_{1 / 2}\right)+$ $\mathrm{H}-\mathrm{CD}_{3}(v=0) \longrightarrow \mathrm{HCl}\left(v^{\prime}=1\right)+\mathrm{CD}_{3}$. Note that $\mathrm{R} 1$, the ground state transition, has much larger contribution to the reaction than either R2 or R3, and hence we multiplied the calculated ICSs for R2 and R3 by factors of 5 and 2 respectively to show their features. Since the ICS for the ground state transition rises before the adiabatic energy barrier for the reaction $(\sim 0.16 \mathrm{eV})$, the quantum tunneling effect is expected to have contribution to the overall reaction. At $\sim 0.4 \mathrm{eV}$, the ICSs for R2 and R3 becomes nonzero, indicating the first excited product channel opens at this energy. Figures 3(b) and (c) present the comparison between the experimental (grey dots) and QRS calculated (black curves) ICSs of R2 and R3 relative to R1. In both cases, very good agreement is observed close to the energetic threshold. The discrepancies found beyond 15kcal/mol for R2/R1 in Figure 3(b) arises from the fact that experiment sampled the product at low rotational states, whereas the QRS results are cumulative cross sections which correspond to reactions from all open channels. We also note that $\mathrm{Cl}\left({ }^{2} \mathrm{P}_{1 / 2}\right)$ is found to have a larger contribution to the $\mathrm{HCl}\left(v^{\prime}=1\right)$ product than $\mathrm{Cl}\left({ }^{2} \mathrm{P}_{3 / 2}\right)$, this is due to the 

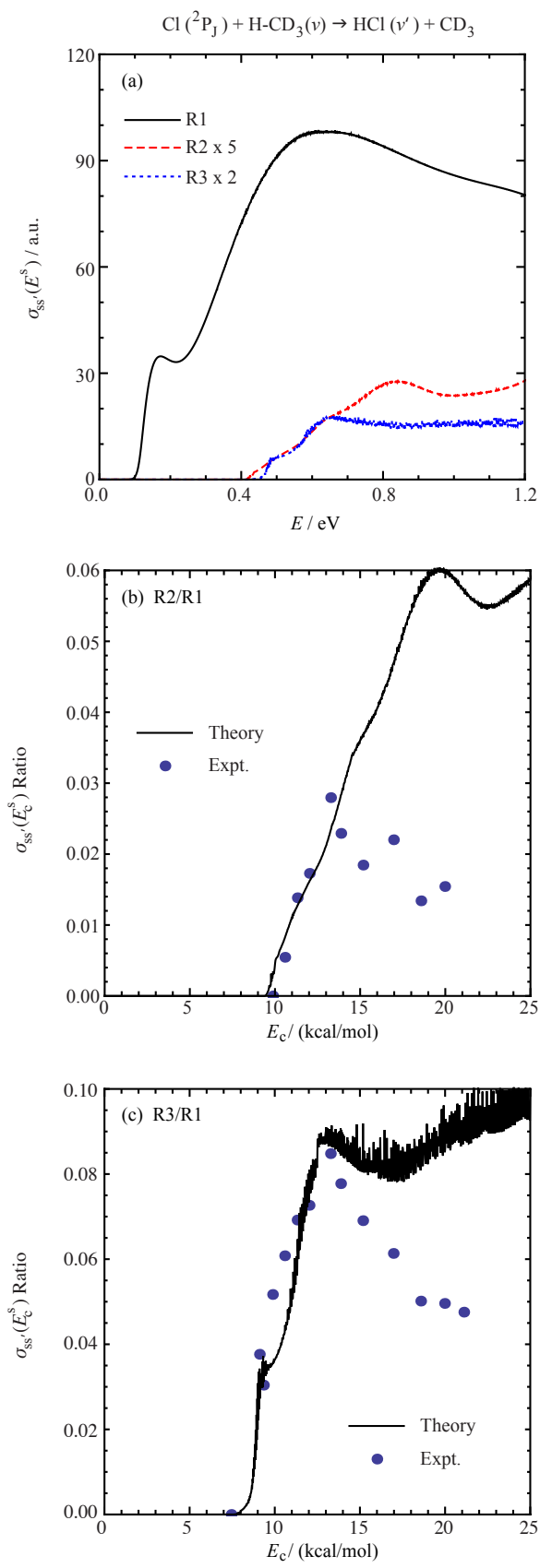

Figure 3: Plot of state-to-state integral cross sections of the $\mathrm{Cl}\left({ }^{2} \mathrm{P}_{\mathrm{J}}\right)+$ $\mathrm{HCD}_{3}(v) \longrightarrow \mathrm{HCl}\left(v^{\prime}\right)+\mathrm{CD}_{3}$ reaction versus collision energy (panel a), and comparisons to experiment of the state-to-state integral cross sections relative to that of the ground state versus collision energy (panels b and c). R1, R2 and R3 refer to $\mathrm{Cl}\left({ }^{2} \mathrm{P}_{3 / 2}\right)(v=0)\left(v^{\prime}=0\right), \mathrm{Cl}\left({ }^{2} \mathrm{P}_{3 / 2}\right)(v=0)\left(v^{\prime}=1\right)$, and $\mathrm{Cl}\left({ }^{2} \mathrm{P}_{1 / 2}\right)(v=0)\left(v^{\prime}=1\right)$, respectively. 
fact that the reactant and product energy levels are nearly equal.

Recently, we extended the application of RD QRS calculation to H-abstraction from cyclic organic molecules, i.e. the $\mathrm{H}+$ cyc $-\mathrm{C}_{3} \mathrm{H}_{6} \longrightarrow \mathrm{H}_{2}+$ cyc- $\mathrm{C}_{3} \mathrm{H}_{5}$ reaction [55]. It is of particular interest because of the unusually strong $\mathrm{C}-\mathrm{H}$ bond [98-100] and because the product cyc $-\mathrm{C}_{3} \mathrm{H}_{5}$ can undergo a ring opening isomerization to form $\mathrm{CH}_{2} \mathrm{CHCH}_{2}$ [101]. This reaction presents an interesting challenge in the PES construction because of the vdW complex in the reactant channel. In Figure 4(a), the dots shows the minimum energy path (MEP) of the reaction in hyperspherical coordinates converted from the conventional Jacobi coordinates, i.e. $R$ is taken as the distance between the CoMs of $\mathrm{H}_{2}$ and $\mathrm{cyc}-\mathrm{C}_{3} \mathrm{H}_{5}$. The black dots were obtained directly from the intrinsic reaction path (IRC) calculation in the MOLPRO program [71], and the solid black curve represents the continuation of the ab initio points. Points at large $\rho$ values are included in the graph as blue dots. The position of the TS and vdW complex structure are highlighted as the red and brown dots, respectively. An interesting feature of the MEP can be found in the reactant channel, near the vdW complex position, that the hyperspherical radius $\rho$ of the points on MEP decreases as the hyperspherical angle $\delta$ increases. The normally observed beharviour of the MEP, shown as the dashed black curve on Figure 4(a), shows a much smoother transition from reactants to products. The reason of this unusual behaviour is due to the structure of the vdW complex, which has the incoming $\mathrm{H}$-atom sitting on top of the cyc- $\mathrm{C}_{3} \mathrm{H}_{6}$ ring. Therefore, around this vdW complex position, even though the $\mathrm{H}-\mathrm{H}$ distance (Figure 1) increases as the MEP is followed towards the reactant channel, the Jacobi coordinate $R$ may still decrease, resulting in a decreasing $\rho$. At further distance, this vdW complex is no 
longer the most stable structure; the $\mathrm{H}-\mathrm{H}-\mathrm{C}$ triatom can exist in a near-collinear geometry, and hence at large $\rho$ values the MEP in the reactant channel follows the usual route. This $\mathrm{H}-\mathrm{H}-\mathrm{C}$ bending mode is not treated explicitly in a 2-D QRS calculation, and since the collinear or near-collinear geometry is broken around the vdW complex, the $(1+1) \mathrm{D}$ method cannot be used here to construct a reliable 2-D PES. Note that if the bending mode is considered so that one would have a $(1+2) \mathrm{D}$ PES, an RD QRS calculation could still be performed using a carefully derived kinetic energy operator, although the mathematics involved in this pseudo-3-D model can be complicated. Alternatively, one can define the $R$ coordinate relative to the $\mathrm{C}$-atom from which the $\mathrm{H}$-atom is abstracted. This technique has been employed previously in other RD QRS studies [25, 52-54]. The resulting MEP in hyperspherical coordinates is shown in Figure 4(b). We can see that the MEP now exhibits normal behaviour, and the vdW complex (brown dot) lies on this path nicely. A total of 163 ab initio data points were calculated in order to fit the 2-D PES. The fitted parameter values as well as the exact 2-D potential function can be found in ref [55].

Here we show in Figure 4(c) and (d) the comparison of our calculated rate constants to the experimental $[98,99]$ and other theoretical [102] results. At 500K, the QRS rate constant is $8.342 \times 10^{-16} \mathrm{~cm}^{3}$ molecule ${ }^{-1} \mathrm{~s}^{-1}$, which is $\sim 5$ times greater than the TST value of $1.604 \times 10^{-16} \mathrm{~cm}^{3}$ molecule ${ }^{-1} \mathrm{~s}^{-1}$. At $300 \mathrm{~K}$, it is approximately 20 times greater. The strong contribution of quantum tunnelling to the reaction rate is clearly observed, especially at lower $T$. We can see that our results exhibit very good agreement to both sets of the experimental data (the red and blue curves). The study by Cohen et al. [102], shown as the brown curves in 
$\mathrm{H}+$ cyc- $\mathrm{C}_{3} \mathrm{H}_{6} \rightarrow \mathrm{H}_{2}+$ cyc- $\mathrm{C}_{3} \mathrm{H}_{5}$
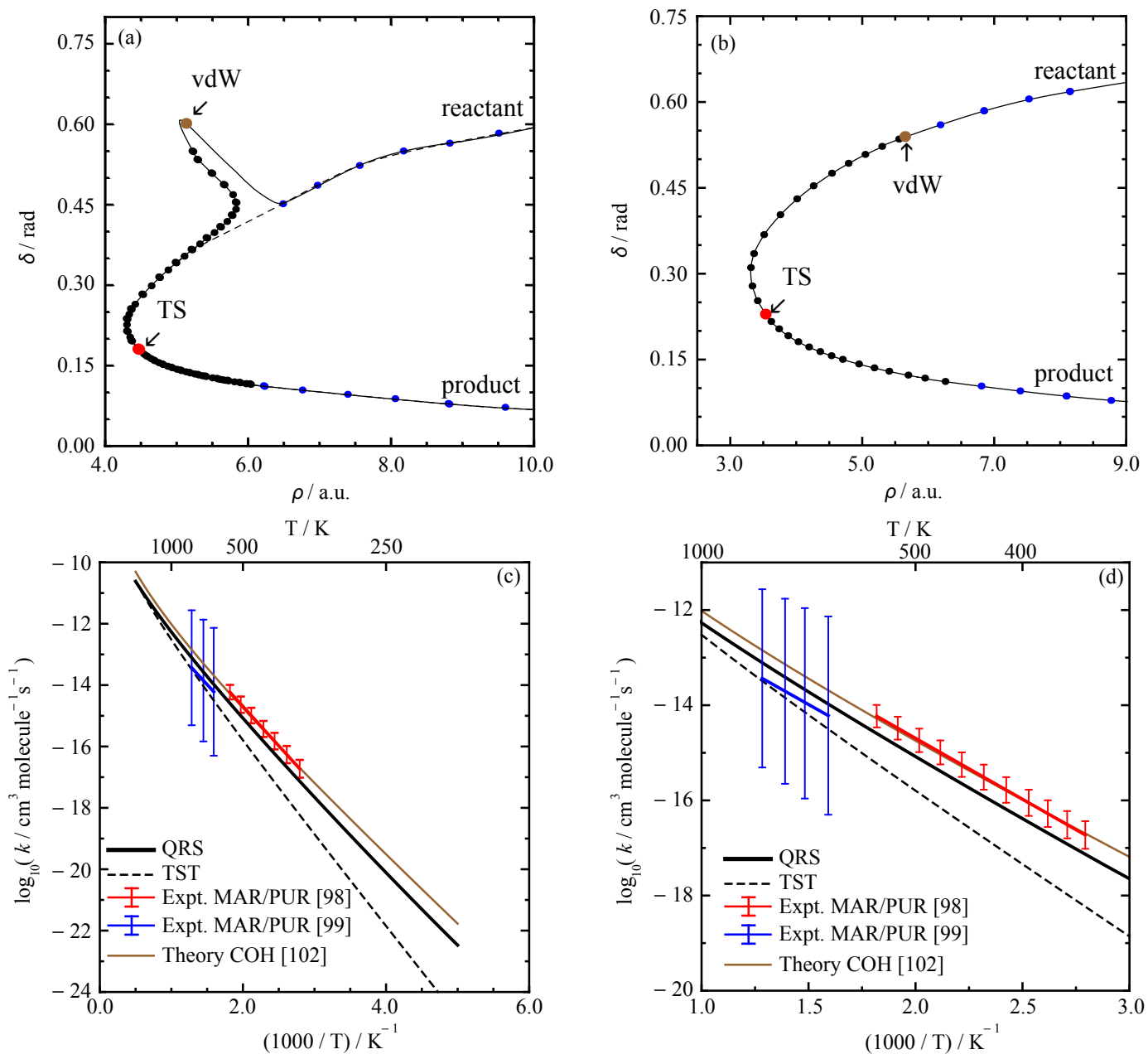

Figure 4: Plots of the minimum energy paths of the $\mathrm{H}+$ cyc- $\mathrm{C}_{3} \mathrm{H}_{6} \longrightarrow \mathrm{H}_{2}+$ cyc $-\mathrm{C}_{3} \mathrm{H}_{5}$ reaction in hyperspherical coordinates converted from (a) conventional Jacobi coordinates and (b) Jacobi coordinates with $R$ defined relative to the $\mathrm{C}$ atom from which the $\mathrm{H}$-atom is abstracted. Comparison of the calculated quantum and transition state theory thermal rate constants with results from the literature for the $\mathrm{H}+$ cyc $-\mathrm{C}_{3} \mathrm{H}_{6} \longrightarrow \mathrm{H}_{2}+$ cyc $-\mathrm{C}_{3} \mathrm{H}_{5}$ reaction at (c) $200 \mathrm{~K}$ to $2000 \mathrm{~K}$ and (d) $333 \mathrm{~K}$ to $1000 \mathrm{~K}$. 
Figure 4, was based on the TST framework, and the values of the parameters in the Arrhenius equation were chosen to fit the lower $T$ experimental data [99]. As a result, it has better agreement to the red curves than our QRS rate constant, but worse agreement to the blue curves.

\section{Semiclassical Transition State Theory}

\subsection{Theoretical Overview}

The SCTST framework is an alternative approach to rate constant calculations that requires significantly less information from the PES. In SCTST, reaction probabilities are calculated from an expression for the system's energy at the transition state (TS). In the most common implementation of SCTST, this expression is derived using second-order vibrational perturbation theory (VPT2) [103], although other higher-order approaches are, in principle, possible [104]. Third and fourth derivatives of the electronic energy are treated as perturbations to a harmonic expansion of the PES and used to calculate a set of anharmonic constants. Reaction probabilities are calculated directly from the harmonic frequencies and anharmonic constants of the TS. SCTST therefore cannot be used to calculate $S$-matrix elements or differential cross sections.

\subsubsection{Anharmonic Parameters}

Formulas for calculating these anharmonic terms within the VPT2 framework at any stationary point (TS or minimum) are presented here. In a FD calculation, diagonal and off-diagonal anharmonic constants $\left(x_{i i}\right.$ and $\left.x_{i k}\right)$ are calculated as 
follows [31, 103]:

$$
x_{i i}=\frac{\hbar^{2}}{16 \omega_{i}^{2}}\left(f_{i i i i}-\sum_{k=1}^{F} \frac{f_{i i k}^{2}}{\omega_{k}^{2}} \frac{8 \omega_{i}^{2}-3 \omega_{k}^{2}}{4 \omega_{i}^{2}-\omega_{k}^{2}}\right)
$$

and

$$
\begin{array}{r}
x_{i k}=\frac{\hbar^{2}}{4 \omega_{i} \omega_{k}}\left(f_{i i k k}-\sum_{j=1}^{F} \frac{f_{i i j} f_{j k k}}{\omega_{j}^{2}}+\sum_{j=1}^{F} \frac{2 f_{i i k}^{2}\left(\omega_{i}^{2}+\omega_{k}^{2}-\omega_{j}^{2}\right)}{\left[\left(\omega_{i}+\omega_{k}\right)^{2}-\omega_{j}^{2}\right]\left[\left(\omega_{i}-\omega_{k}\right)^{2}-\omega_{j}^{2}\right]}\right)+ \\
\left(\frac{\omega_{i}}{\omega_{k}}+\frac{\omega_{k}}{\omega_{i}}\right) \sum_{\alpha} B_{\alpha}\left(\zeta_{i k}^{\alpha}\right)^{2}, i \neq k, \quad
\end{array}
$$

where $\omega_{i}$ is the harmonic frequency for the $i^{\text {th }}$ vibrational mode, and $f_{i j k}$ and $f_{i i j j}$ denote third and fourth potential derivatives along normal mode eigenvectors. $F$ denotes the number of vibrational degrees of freedom (DOFs) of the species. The last term in the expression for off-diagonal anharmonic constants treats coupling between vibrational and rotational DOFs. $B_{\alpha}$ represents a rotational constant, and $\zeta_{i k}^{\alpha}$ is a Coriolis coupling constant [105]. Additionally, a constant energy parameter $G_{0}$ arises from the VPT2 treatment [106]:

$$
\begin{aligned}
G_{0}=\frac{\hbar^{2}}{64} \sum_{i=1}^{F} \frac{f_{i i i i}}{\omega_{i}^{2}}-\frac{7 \hbar^{2}}{576} \sum_{i=1}^{F} \frac{f_{i i i}^{2}}{\omega_{i}^{2}} & +\frac{3 \hbar^{2}}{64} \sum_{i \neq k} \frac{f_{i i k}^{2}}{\left(4 \omega_{i}^{2}-\omega_{k}^{2}\right) \omega_{i}^{2}} \\
& -\frac{\hbar^{2}}{4} \sum_{i<j<k} \frac{f_{i j k}^{2}}{\left[\left(\omega_{i}+\omega_{k}\right)^{2}-\omega_{j}^{2}\right]\left[\left(\omega_{i}-\omega_{k}\right)^{2}-\omega_{j}^{2}\right]} .
\end{aligned}
$$

In an RD SCTST calculation, anharmonic constants are calculated only for a subset of vibrational modes of the TS. These modes will be referred to as the "active modes," and the reaction mode must be included in this set. In this case, only the active modes are considered in the above sums. For example, the diagonal anharmonic constant for the reaction mode in a 1-D calculation is given as follows:

$$
x_{F F}=\frac{\hbar^{2}}{16 \omega_{F}^{2}}\left(f_{F F F F}-\frac{5 f_{F F F}^{2}}{3 \omega_{F}^{2}}\right)
$$


where $F$ denotes the index of the reaction mode. In the remainder of this section, $d$ will be used to denote the number of modes considered in calculations, such that $d=F$ for $\mathrm{FD}$ calculations, and $d<F$ for RD calculations. Indices are ordered such that the $F^{\text {th }}$ mode is the reaction mode, and the last $d$ modes, with indices $F-d+1$ to $F$, are the active modes. The remaining modes, with indices 1 to $F-d$, are spectator modes, defined as in Section 2.1.2.

\subsubsection{State-Dependent Reaction Probability}

If more than one mode is considered, the reaction probability is calculated for each configuration $\{n\}$ of the quantum numbers of the $d-1$ non-reactive active modes using WKB Theory [107]. The WKB integral for each configuration is calculated as follows in a conventional SCTST treatment if the "deep tunnneling" correction is not applied [31]:

$$
\theta_{\{n\}}\left(E_{\mathrm{v}}\right)=\pi \frac{-\Omega_{\{n\}}+\left[\Omega_{\{n\}}^{2}+4 x_{F F}\left(\Delta V_{f}^{\ddagger}+G_{0}+E_{\{n\}}-E_{\mathrm{v}}\right)\right]^{1 / 2}}{2 x_{F F}},
$$

where $E_{\mathrm{v}}$ represents the energy in the active vibrational DOFs of the TS, $\Delta V_{f}^{\ddagger}$ denotes the reaction's forward barrier height, and $x_{F F}$ denotes the diagonal anharmonic constant for the reaction mode. $E_{\{n\}}$ represents the energy in the nonreactive active modes, which is given in the VPT2 framework as follows:

$$
E_{\{n\}}=\sum_{i=F-d+1}^{F-1} \hbar \omega_{i}\left(n_{i}+1 / 2\right)+\sum_{i=F-d+1}^{F-1} \sum_{k=i}^{F-1} x_{i k}\left(n_{i}+1 / 2\right)\left(n_{k}+1 / 2\right) .
$$

$\Omega_{\{n\}}$ represents an effective reaction mode frequency that accounts for coupling between other active modes and the reaction mode. It is calculated as follows:

$$
\Omega_{\{n\}}=\operatorname{Im}\left[\hbar \omega_{F}+\sum_{i=F-d+1}^{F-1} x_{i F}\left(n_{i}+\frac{1}{2}\right)\right] .
$$


This WKB integral is equivalent to that calculated at energy $E_{\mathrm{v}}-E_{\{n\}}$ for a symmetric Eckart potential defined as follows [108, 109]:

$$
V_{\mathrm{se}}(s)=\Delta V_{f}^{\ddagger}+G_{0}-D_{\mathrm{se}}+4 D_{\mathrm{se}} \frac{\exp (\alpha s)}{[1+\exp (\alpha s)]^{2}},
$$

where

$$
D_{\mathrm{se}}=-\frac{\Omega_{\{n\}}^{2}}{4 x_{F F}}
$$

and

$$
\alpha=\frac{\left(-8 x_{F F}\right)^{1 / 2}}{\hbar}
$$

with $s$ as the reaction coordinate, with units (mass ${ }^{1 / 2}$ length). The state-dependent reaction probability is calculated from the WKB integral as follows [31]:

$$
P_{\{n\}}\left(E_{\mathrm{v}}\right)=\left\{1+\exp \left[2 \theta_{\{n\}}\left(E_{\mathrm{v}}\right)\right]\right\}^{-1}
$$

This method for calculating reaction probabilities and associated rate constants will be referred to as SCTST-M.

The reactant and product asymptotes of the symmetric Eckart potential given above do not necessarily correspond to the reactant or product energies and are instead given as follows:

$$
\lim _{s \rightarrow \pm \infty} V_{\mathrm{se}}(s)=\Delta V_{f}^{\ddagger}-D_{\mathrm{se}}
$$

Recently, Wagner [108] proposed a correction to SCTST that rectifies this issue. A piecewise asymmetric Eckart potential is constructed such that its asymptotes are the correct reactant and product energies and its second, third, and fourth derivatives at the TS match those of the symmetric Eckart potential defined above. Details of the construction of this potential can be found in the Supplementary 
Information of ref [108]. Its WKB integral can be calculated analytically [108], and the associated state-dependent reaction probability is calculated as described in previous studies $[35,110]$. This approach to SCTST calculations will be referred to as SCTST-W.

In a 1-D calculation, the reaction probability is calculated as described above with $E_{\{n\}}=0$ and $\Omega_{\{n\}}=\operatorname{Im}\left(\hbar \omega_{F}\right)$.

\subsubsection{Cumulative Reaction Probability}

For calculations involving more than one DOF, the cumulative reaction probability (CRP), $N\left(E_{\mathrm{v}}\right)$, is defined as a sum over all energetically allowed configurations of $\{n\}$ at energy $E_{\mathrm{v}}[29-31,61,111]$ :

$$
N\left(E_{\mathrm{v}}\right)=\sum_{\{n\}} P_{\{n\}}\left(E_{\mathrm{v}}\right)
$$

This is calculated most accurately by enumerating over all configurations. However, this can be computationally very expensive if more than 3 or 4 DOFs are considered in the calculation. In this case, the Wang-Landau algorithm [112] can be applied to obtain an approximation to the CRP at a reduced computational cost. Briefly, this involves performing a random walk in the configuration space of $\{n\}$. The range of $E_{\mathrm{v}}$ considered in the calculation is partitioned into a number of bins, and the density of states in each bin, $\rho\left(E_{i}\right)$, is approximated from the number of states sampled in that bin during the random walk. Approximate values of $\Omega_{\{n\}}$ and $E_{\{n\}}$ for each bin are obtained by averaging those of all states sampled within each bin $[113,114]$. These are used to calculate the average reaction probability, $P_{i}(E)$, for each bin. With this approximation, the CRP is calculated as follows 
[114]:

$$
N(E)=\sum_{i=1}^{E / \Delta E} \rho_{\mathrm{TS}}\left(E_{i}\right) P_{i}(E) \Delta E,
$$

where $i$ denotes the bin index.

Rate constants are calculated from the CRP as described in Eq. 12. Note that this algorithm is also useful for calculating the density of states of reactant species within the VPT2 framework, which can be used to calculate anharmonic partition functions.

\subsection{Rate Constants from Potential Energy Surfaces \\ 3.2.1 Force Constant Calculations}

This section describes how to calculate the parameters required for an SCTST calculation from a PES defined in hyperspherical coordinates, $V(\rho, \delta)$. Frequencies and anharmonic constants were calculated at the saddle point of the PES instead of the TS geometry obtained from ab initio calculations. Derivatives with respect to Jacobi coordinates $R$ and $r$ can be calculated using the chain rule as follows:

$$
\begin{gathered}
\frac{\partial}{\partial r}=\left(\frac{m_{2}}{\mu}\right)^{1 / 2}\left(\sin \delta \frac{\partial}{\partial \rho}+\frac{\cos \delta}{\rho} \frac{\partial}{\partial \delta}\right) \\
\frac{\partial}{\partial R}=\left(\frac{m_{1}}{\mu}\right)^{1 / 2}\left(\cos \delta \frac{\partial}{\partial \rho}-\frac{\sin \delta}{\rho} \frac{\partial}{\partial \delta}\right),
\end{gathered}
$$

where $m_{1}, m_{2}$, and $\mu$ are defined as in Eq. 15. The mass-weighted Hessian matrix is given in Jacobi coordinates as follows [115]:

$$
H=\left(\begin{array}{cc}
m_{1}^{-1} \frac{\partial^{2} V}{\partial^{2} R} & \left(m_{1} m_{2}\right)^{-1 / 2} \frac{\partial^{2} V}{\partial R \partial r} \\
\left(m_{1} m_{2}\right)^{-1 / 2} \frac{\partial^{2} V}{\partial R \partial r} & m_{2}^{-1} \frac{\partial^{2} V}{\partial^{2} r}
\end{array}\right) .
$$


Diagonalizing this matrix yields two frequencies, one real and one imaginary. Derivatives along the eigenvectors $\left\{\mathbf{v}_{i}\right\}$ can be calculated analytically as follows:

$$
\frac{\partial}{\partial q_{i}}=\mathbf{v}_{i} \cdot\left(m_{1}^{-1 / 2} \frac{\partial}{\partial R}, m_{2}^{-1 / 2} \frac{\partial}{\partial r}\right)
$$

where $q_{i}$ denotes the mass-weighted normal mode coordinate associated with mode $i$.

\subsubsection{Barrier Height Calculations}

The forward barrier height of the reaction is calculated from the PES as follows:

$$
\Delta V_{f}=V\left(\rho_{\mathrm{TS}}, \delta_{\mathrm{TS}}\right)-V\left(\rho_{\max }, \delta_{r}\right)-\frac{\hbar}{2}\left(\left.\frac{m_{\mathrm{H}}+m_{\mathrm{Z}}}{m_{\mathrm{H}} m_{\mathrm{Z}}} \frac{\partial^{2} V}{\partial R^{2}}\right|_{\rho=\rho_{\max }, \delta=\delta_{r}}\right)^{1 / 2}
$$

where $\rho_{\max }$ is the maximum value of $\rho$ considered in QRS calculations, and $\delta_{r}$ is the value of $\delta$ at the minimum of the reactant well at $\rho=\rho_{\max }$. The last term in this equation represents the zero-point energy (ZPE) of the reactant $\mathrm{C}-\mathrm{H}$ bond and must be included because ZPE is not included in the vibrational component of the reactant partition function. The ZPE of the other reactant vibrational modes are included in the PES. The reaction's reverse barrier height is calculated similarly as follows:

$$
\Delta V_{r}=V\left(\rho_{\mathrm{TS}}, \delta_{\mathrm{TS}}\right)-V\left(\rho_{\max }, \delta_{p}\right)-\frac{\hbar}{2}\left(\left.\frac{m_{\mathrm{H}}+m_{\mathrm{Y}}}{m_{\mathrm{H}} m_{\mathrm{Y}}} \frac{\partial^{2} V}{\partial r_{1}^{2}}\right|_{\rho=\rho_{\max }, \delta=\delta_{p}}\right)^{1 / 2} .
$$

The derivative with respect to $r_{1}$, the product bond length, is calculated based on the assumption that the TS is collinear. Because the ZPE of the TS is included in $E_{\{n\}}$ (Eq. 23), it is not included when calculating the barrier heights. 

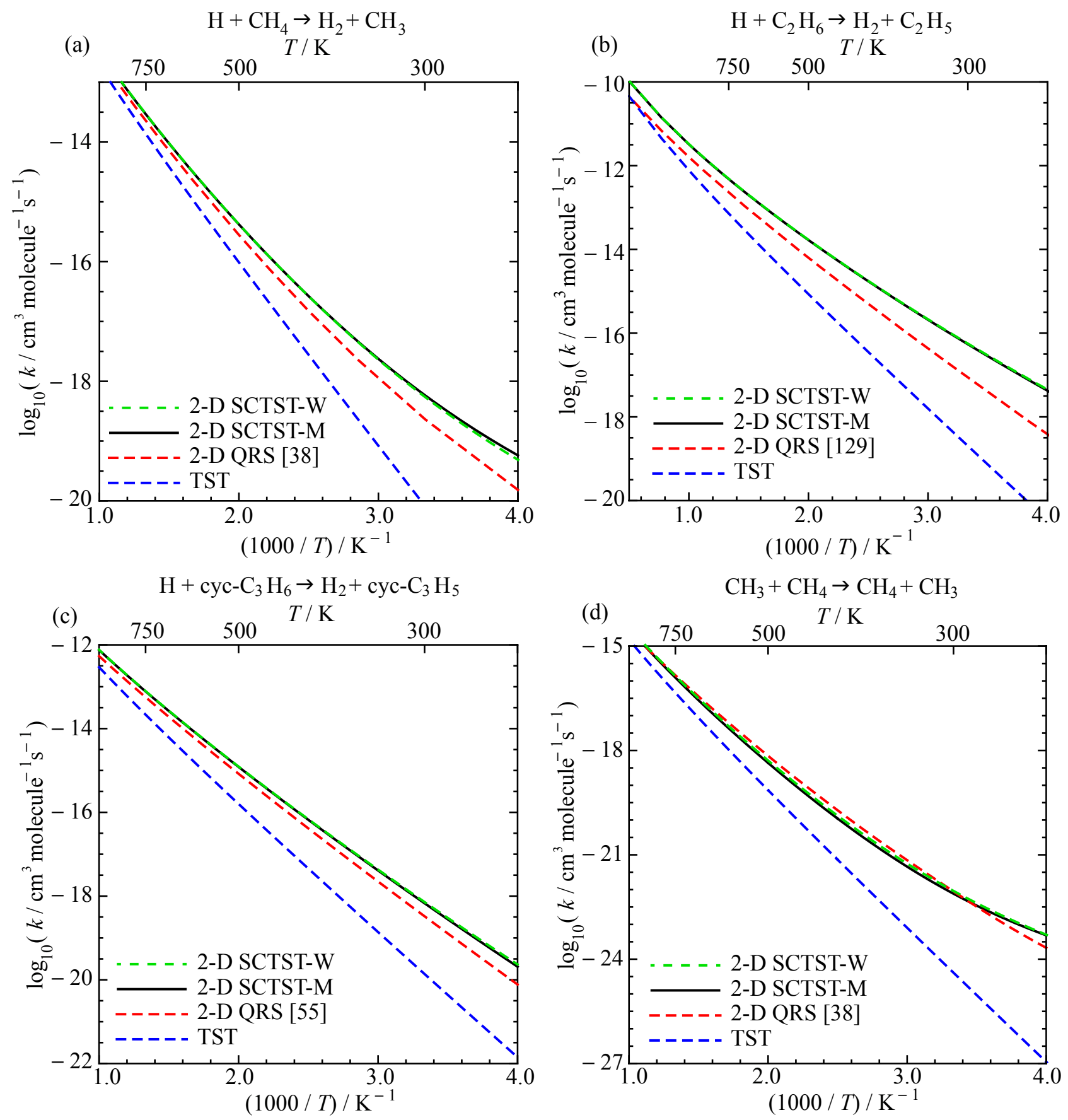

Figure 5: Rate constants calculated from 2-D PESs using SCTST with and without deep tunneling corrections (dashed green and black curves, respectively) presented in comparison to results from TST (dashed blue line) and QRS calculations on the same PES (dashed red line) for four reactions. 


\subsubsection{Results}

Rate constants calculated by applying 2-D SCTST to these PESs are presented in Figure 5 for four reactions: $\mathrm{H}+\mathrm{CH}_{4} \longrightarrow \mathrm{H}_{2}+\mathrm{CH}_{3}, \mathrm{H}+\mathrm{C}_{2} \mathrm{H}_{6} \longrightarrow \mathrm{H}_{2}+$ $\mathrm{C}_{2} \mathrm{H}_{5}, \mathrm{H}+\mathrm{cyc}-\mathrm{C}_{3} \mathrm{H}_{6} \longrightarrow \mathrm{H}_{2}+\mathrm{cyc}-\mathrm{C}_{3} \mathrm{H}_{5}$, and $\mathrm{CH}_{3}+\mathrm{CH}_{4} \longrightarrow \mathrm{CH}_{4}+\mathrm{CH}_{3}$. For all reactions except $\mathrm{H}+\mathrm{C}_{2} \mathrm{H}_{6}$, rate constants calculated using the 2-D SCTST-M method differed from 2-D QRS rate constants by less than a factor of 4 at $250 \mathrm{~K}$. Rate constants for the $\mathrm{H}+\mathrm{C}_{2} \mathrm{H}_{6}$ reaction differed by a factor of 9.67 at $250 \mathrm{~K}$, likely due to the fact that the QRS and SCTST methods are sensitive to different regions of the PES, as discussed previously [115]. Furthermore, applying deep tunneling corrections was found not to significantly affect the calculation of rate constants in the 2-D SCTST framework. Differences between the 2-D SCTST-M and 2-D SCTST-W rate constants are less than $40 \%$ at $250 \mathrm{~K}$ for all four reactions.

Treating only one DOF rather than two was found to yield acceptable approximations of 2-D rate constants. For all reactions except $\mathrm{CH}_{3}+\mathrm{CH}_{4}$, rate constants calculated using the 1-D SCTST-M method differed from their 1-D counterparts by less than a factor of 2 at $250 \mathrm{~K}$ (Figure 6). These rate constants differed by a factor of 6 at $250 \mathrm{~K}$ for the $\mathrm{CH}_{3}+\mathrm{CH}_{4}$ reaction, but applying deep tunneling corrections resulted in agreement to within a factor of 2 at $250 \mathrm{~K}$. Because applying deep tunneling corrections requires marginal additional computational expense relative to the SCTST-M method and yields results of comparable or better accuracy, it was concluded that it is always desirable to apply these corrections. 

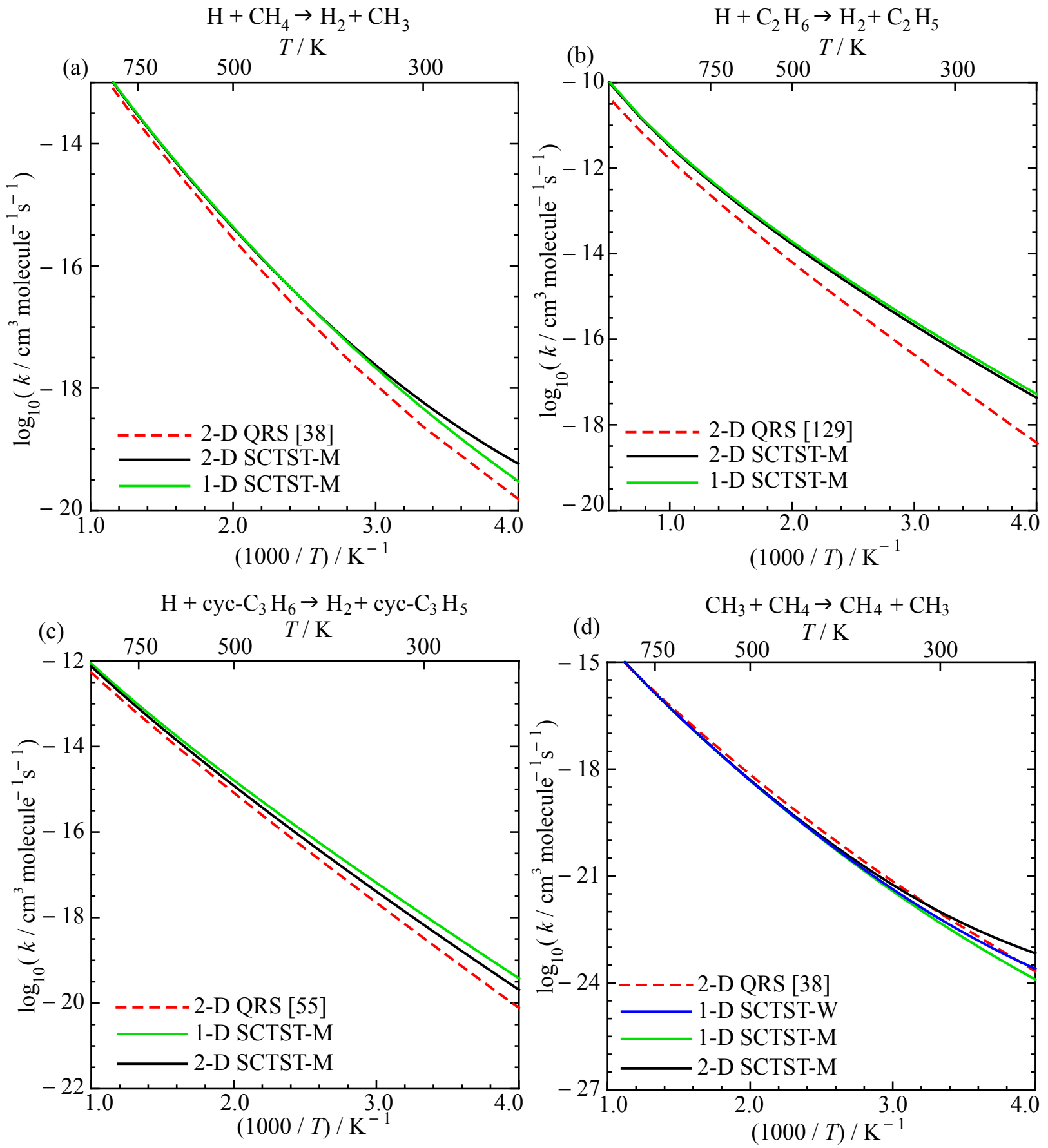

Figure 6: Rate constants calculated using 1-D SCTST methods presented in comparison to 2-D rate constants. 


\subsection{Rate Constants from Ab Initio Calculations}

\subsubsection{Richardson Extrapolation}

Richardson extrapolation can be applied to calculations of numerical derivatives in order to reduce the error associated with higher-order derivatives [116-118]. We applied this method to calculate the third and fourth PES derivatives required for FD and 1-D SCTST calculations [119]. Briefly, a particular numerical derivative is calculated with step sizes $h$ and $c h$, with $c>1$. The values of these derivatives will be denoted $A(h)$ and $A(c h)$, respectively. They can be combined as follows to yield an estimate of the derivative with a lower error:

$$
B(h)=\frac{c^{2} A(h)-A(c h)}{c^{2}-1} .
$$

$B(h)$ is a "first-order extrapolation" of the derivative in question. Two first-order extrapolations with different step sizes can be combined to yield a "second-order extrapolation" of the derivative, $C(h)$ :

$$
C(h)=\frac{c^{4} B(h)-B(c h)}{c^{4}-1}=\frac{c^{6} A(h)-\left(c^{2}+c^{4}\right) A(c h)+A\left(c^{2} h\right)}{\left(c^{2}-1\right)^{2}\left(c^{2}+1\right)} .
$$

This involves calculating zeroth-order derivatives at three different step sizes, each differing by a factor of $c$. This approach can be applied multiple times to yield successively higher-order extrapolations of the derivative with successively smaller errors.

\subsubsection{Force Constant Calculations}

Numerical calculations of the third and fourth derivatives (force constants) required in SCTST involve calculating the Hessian matrix at positive and negative 
displacements along each normal mode eigenvector [120-122]. First, the massweighted Hessian matrix at the equilibrium geometry, $\mathbf{M H}(0) \mathbf{M}$, is diagonalized to yield a matrix $\mathbf{L}$ of eigenvectors. $\mathbf{M}$ is defined as follows:

$$
M_{i j}=\delta_{i j} m_{i}^{-1 / 2}
$$

with each mass $m_{i}$ associated with a Cartesian coordinate for an atom. The matrix $\boldsymbol{\Phi}(0)$ represents the diagonal matrix of eigenvalues:

$$
\Phi(0)=\mathbf{L}^{\mathrm{T}} \mathbf{M H}(0) \mathbf{M L} .
$$

$\Phi$ is also defined at positive and negative displacements along eigenvectors, $\pm \Delta Q_{i}$. In these cases, it is not necessarily diagonal:

$$
\Phi\left( \pm \Delta Q_{i}\right)=\mathbf{L}^{\mathrm{T}} \mathbf{M H}\left( \pm \Delta Q_{i}\right) \mathbf{M L}
$$

Numerically differentiating the elements of $\boldsymbol{\Phi}$ yields third derivatives $f_{i j k}$ as follows $[120,121]:$

$f_{i j k}=\frac{1}{3}\left(\frac{\boldsymbol{\Phi}_{j k}\left(\Delta Q_{i}\right)-\boldsymbol{\Phi}_{j k}\left(-\Delta Q_{i}\right)}{2 \Delta Q_{i}}+\frac{\boldsymbol{\Phi}_{k i}\left(\Delta Q_{j}\right)-\boldsymbol{\Phi}_{k i}\left(-\Delta Q_{j}\right)}{2 \Delta Q_{j}}+\frac{\boldsymbol{\Phi}_{i j}\left(\Delta Q_{k}\right)-\boldsymbol{\Phi}_{i j}\left(-\Delta Q_{k}\right)}{2 \Delta Q_{k}}\right)$,

and fourth derivatives $f_{i i j j}$ as follows:

$f_{i i j j}=\frac{1}{2}\left(\frac{\boldsymbol{\Phi}_{i i}\left(\Delta Q_{j}\right)+\boldsymbol{\Phi}_{i i}\left(-\Delta Q_{j}\right)-2 \boldsymbol{\Phi}_{i i}(0)}{\Delta Q_{j}^{2}}+\frac{\boldsymbol{\Phi}_{j j}\left(\Delta Q_{i}\right)+\boldsymbol{\Phi}_{j j}\left(-\Delta Q_{i}\right)-2 \boldsymbol{\Phi}_{j j}(0)}{\Delta Q_{i}^{2}}\right)$.

Note that Richardson extrapolation can be applied independently to the calculation of Hessian matrix elements and to the calculation of derivatives of the elements of $\Phi$. 

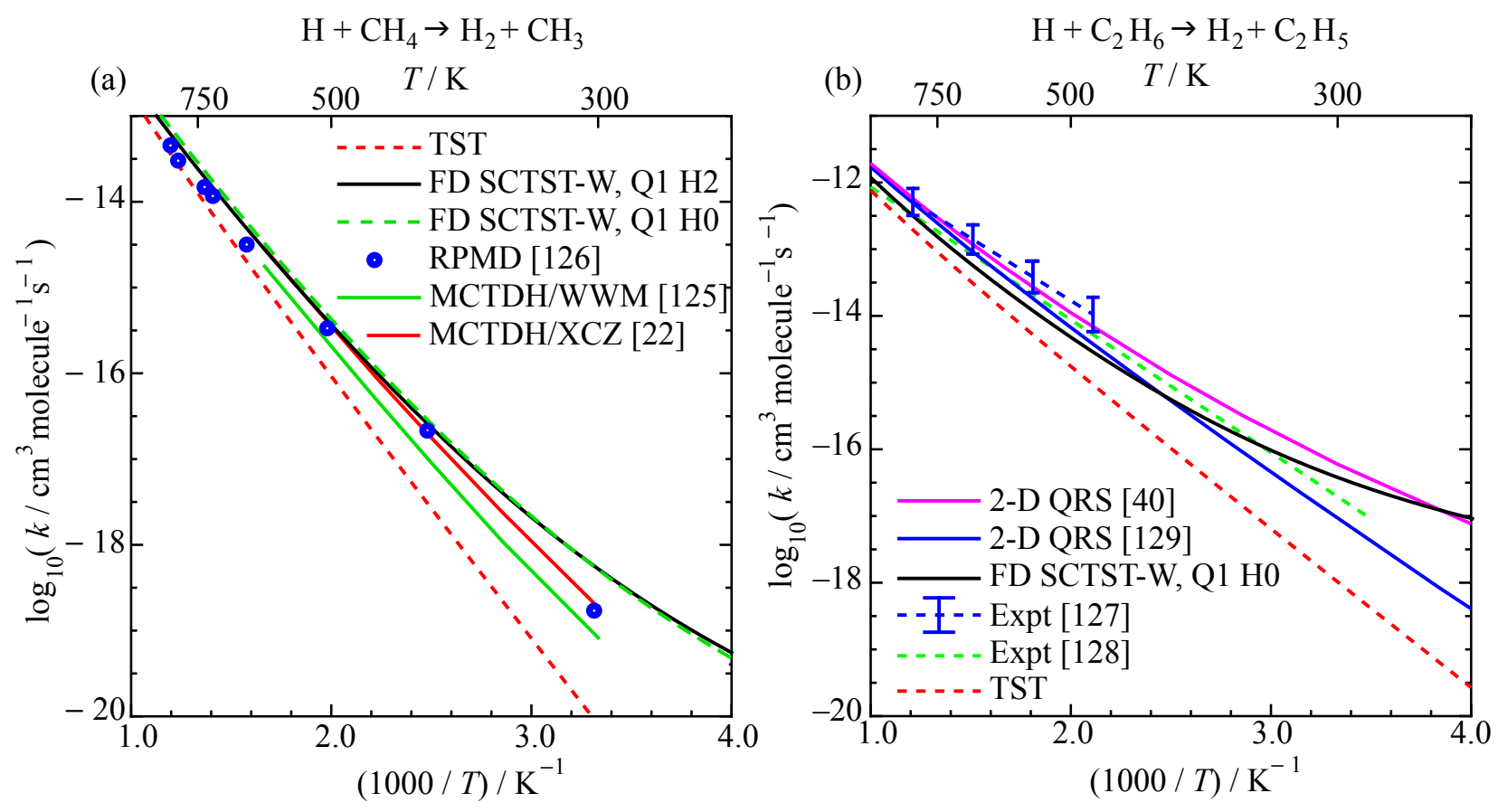

Figure 7: Rate constants for the $\mathrm{H}+\mathrm{CH}_{4} \longrightarrow \mathrm{H}_{2}+\mathrm{CH}_{3}$ and $\mathrm{H}+\mathrm{C}_{2} \mathrm{H}_{6} \longrightarrow \mathrm{H}_{2}+$ $\mathrm{C}_{2} \mathrm{H}_{5}$ reactions calculated using the FD SCTST-W method from PES derivatives obtained from ab initio calculations, presented in comparison to results from other theoretical and experimental studies.

The step size used in VPT2 calculations is often specified in dimensionless reduced normal coordinates $[121,123,124]$. The displacement along each eigenvector, $\Delta Q_{i}$, with units (length mass ${ }^{-1 / 2}$ ), is calculated from a displacement $\Delta q$ in reduced normal coordinates as follows:

$$
\Delta Q_{i}=\frac{\hbar^{1 / 2}}{\omega_{i}^{1 / 2}} \Delta q
$$

\subsubsection{Results}

Previously [119], we used these $a b$ initio methods to calculate the force constants required for SCTST calculations for two reactions $\left(\mathrm{H}+\mathrm{CH}_{4} \longrightarrow \mathrm{H}_{2}+\mathrm{CH}_{3}\right.$ and $\mathrm{H}+\mathrm{C}_{2} \mathrm{H}_{6} \longrightarrow \mathrm{H}_{2}+\mathrm{C}_{2} \mathrm{H}_{5}$ ). This enabled a comparison of results from the FD and 
1-D SCTST methods. FD results, calculated with deep tunneling corrections, are presented in Figure 7. Calculations performed at two different levels of accuracy are shown for the $\mathrm{H}+\mathrm{CH}_{4}$ reaction. In both of these calculations, fourth-order Richardson extrapolation was first applied to calculate Hessian matrix elements of the TS, which were used to calculate frequencies and normal mode eigenvectors. At the higher level of accuracy, "Q1 H2," second-order Richardson extrapolation was applied to the calculation of Hessian matrix elements at displaced geometries, which requires three Hessian matrix calculations with different step sizes at each geometry. The resulting converged Hessian matrix elements were then used to calculate first-order derivatives of matrix elements along normal modes. In total, this method required 149 TS Hessian matrix calculations. The rate constants from this Q1 H2 calculation exhibit acceptable agreement with those from other FD theoretical studies. They differ from those of two previous MCTDH calculations $[22,125]$ by factors of 2.75 and 6.23 at $300 \mathrm{~K}$, and from those from an RPMD calculation [126] by a factor of 3.08 at $300 \mathrm{~K}$.

At the lower level of accuracy, "Q1 H0," Richardson extrapolation was not applied to the calculation of Hessian matrix elements at displaced geometries, and first-order Richardson extrapolation was applied to the differentiation of Hessian matrix elements. This method required only 53 TS Hessian matrix calculations and yielded results in good agreement with those from the Q1 H2 method. Rate constants from both methods differed by $14 \%$ at $250 \mathrm{~K}$ (Figure 7a). Additionally, it was found that calculating force constants without applying Richardson extrapolation to differentiation along normal modes did not yield results of sufficient accuracy [119]. 
Because the Q1 H0 method was found to yield results of comparable accuracy to those from the Q1 H2 method at a significantly reduced computational cost, this lower level of accuracy was used in FD SCTST calculations for the $\mathrm{H}+\mathrm{C}_{2} \mathrm{H}_{6}$ reaction. The resulting rate constants are presented in Figure $7 \mathrm{~b}$ with results from other experimental $[127,128]$ and theoretical studies. At $250 \mathrm{~K}$, results from SCTST differ from those from two previous 2-D QRS studies [40, 129] by factors of 1.16 and 14.1, respectively. In the later of these two studies [40], ab initio calculations were performed at a higher level of theory, and better projection methods were used, suggesting that these results are more accurate. At higher temperatures, agreement between these results and those from FD SCTST is not as close but nonetheless acceptable.

Results from 1-D calculations for both reactions are presented in Figure 8. PES derivatives along the reaction mode from the Q1 H2 and Q1 H0 calculations were used to calculate 1-D SCTST-W rate constants for the $\mathrm{H}+\mathrm{CH}_{4}$ and $\mathrm{H}+$ $\mathrm{C}_{2} \mathrm{H}_{6}$ reactions, respectively. These rate constants are labeled "1-D SCTST-W, Hessians" in Figure 8 and differ from FD rate constants by factors of 4.54 and 2.14 at $250 \mathrm{~K}$ for the $\mathrm{H}+\mathrm{CH}_{4}$ and $\mathrm{H}+\mathrm{C}_{2} \mathrm{H}_{6}$ reactions, respectively. Because the FD and 1-D calculations were performed at the same level of accuracy for each reaction, this comparison enables a direct evaluation of the effect of reducing the number of DOFs treated in an SCTST calculation.

The force constants required for a 1-D calculation were also calculated by differentiating single-point energies calculated along the reaction mode, which is more computationally efficient than differentiating Hessian matrix elements. Results from these calculations are labeled "1-D SCTST-W, energies" in Figure 8. These 

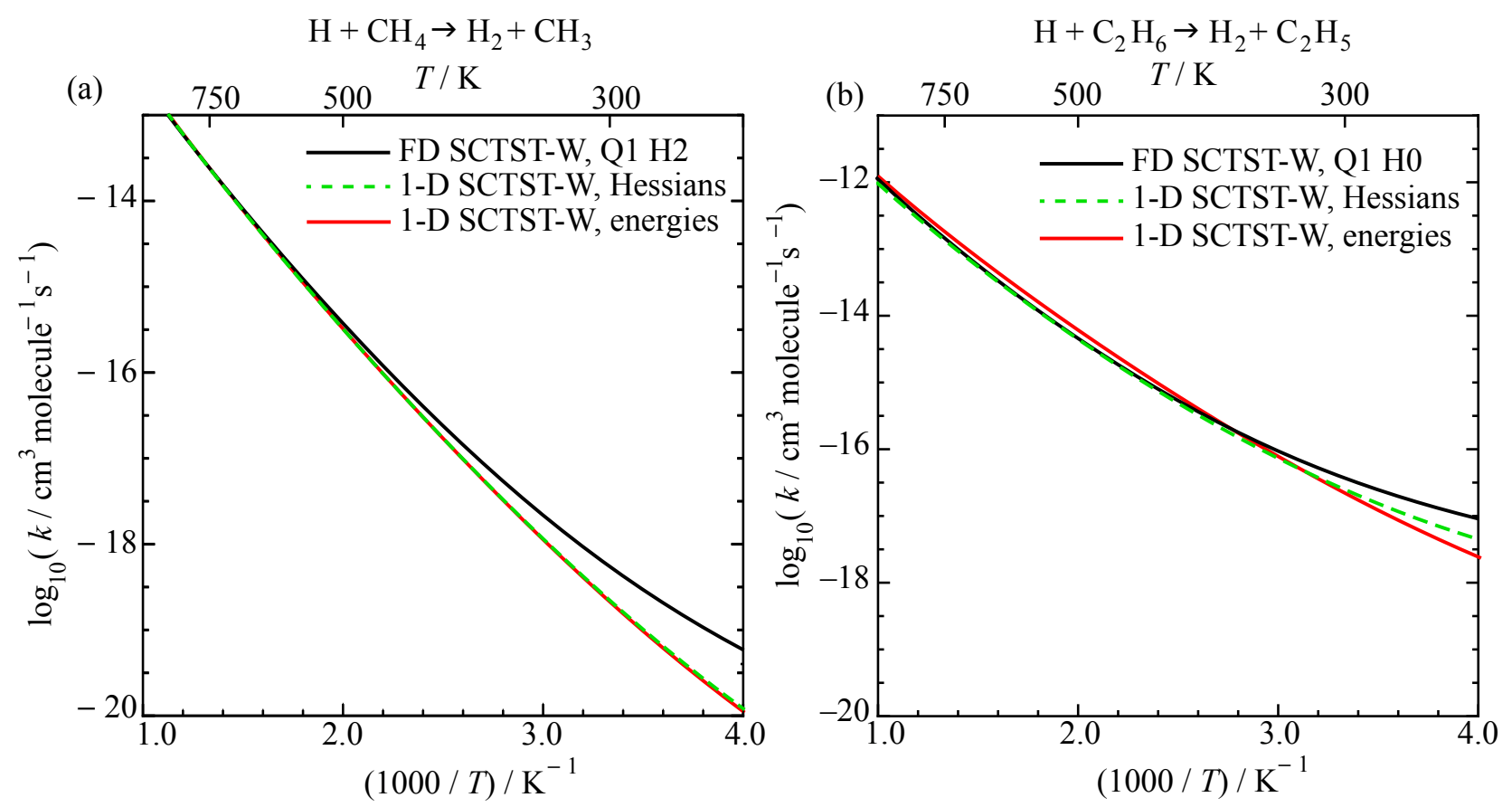

Figure 8: Rate constants for the $\mathrm{H}+\mathrm{CH}_{4}$ and $\mathrm{H}+\mathrm{C}_{2} \mathrm{H}_{6}$ reactions calculated using the 1-D SCTST-W method presented in comparison to FD results. Results labeled "Hessians" were calculated using force constants obtained by differentiating Hessian matrix elements, and those labeled "energies" were obtained by differentiating single-point energies. 
results agreed with those from the "Hessians" calculations to within a factor of 2 at $250 \mathrm{~K}$ for both reactions. In terms of the calculations beyond those required for a conventional TST calculation, this 1-D method is approximately 4 orders of magnitude faster than the implementations of the FD method considered in this study. The computational efficiency of this method and the reasonable level agreement between FD and 1-D results suggest the potential applicability of the 1-D SCTST method to systems too large to be treated with other rate constant theories.

\section{Conclusions}

The reduced-dimensionality procedures have particular advantages in applications to chemical reactions of polyatomic molecules. They make quantum reactive scattering calculations feasible for many new reactions and allow for suitable potential energy surfaces to be produced with minimal computational expense. Semiclassical Transition State Theory brings this computational expense to a minimum while retaining a good accuracy in the prediction of reaction rates. The approach also allows for going directly from a minimum number of ab initio calculations straight to a rate constant.

An advantage of having the quantum dynamics results even in reduced dimensions is that they enable the accuracy of more approximate theories such as SCTST to be evaluated with identical potential energy surfaces. The quantum reactive scattering calculations also provide more detailed results such as cross sections selected in some quantum states of reactants or products that can be compared directly with reaction dynamics experiments. 
The examples chosen here for testing these approaches are reactions of hydrocarbon molecules. However, we see no reason why the methods cannot be applied and adapted to more complex systems. Reduced dimensionality quantum dynamics have been applied to reactions on solid surfaces [130] with approximate potential energy surfaces and, given the major advances being made in accurate quantum chemistry methods, the application of RD SCTST to make reliable predictions on more complex systems such as reactions important in biology or in condensed phases will become computationally viable.

\section{Personal Note on John Light (David Clary)}

In 1977 I started a postdoctoral fellowship working with Robert K. Nesbet at the IBM Research Laboratory, San Jose, California. I was given a program developed by John Light's group to perform $R$-matrix propagator calculations on the $\mathrm{H}+\mathrm{H}_{2}$ reaction in three dimensions. I learnt from this code how to do quantum reactive scattering calculations [131]. Then in 1984, after I had started my own research group at Cambridge University, I initiated a conference on quantum reactive scattering which has turned into a regular international series. I met John Light several times at these conferences and it was an inspiration to hear about his latest research. John was always willing to share his ideas with other researchers. His hallmark was the development of innovative and powerful algorithms that enable new types of calculations to be carried out on molecular collisions and reactions.

\section{Acknowledgements}


X. Shan and D. C. Clary acknowledge financial support from the Leverhulme Trust (Project Grant No. RPG-2013-321). S. M. Greene acknowledges financial support from the Rhodes Trust through a Rhodes Scholarship for graduate study.

\section{References}

[1] E. M. Mortensen, J. Chem. Phys., 48, 4029 (1968).

[2] E. A. McCullough and R. E. Wyatt, J. Chem. Phys., 51, 1253 (1969).

[3] D. G. Truhlar and A. Kuppermann, J. Chem. Phys., 52, 3841 (1970).

[4] G. C. Schatz, J. M. Bowman, and A. Kuppermann, J. Chem. Phys., 58, 4023 (1973).

[5] J. N. L. Connor, W. Jakubetz, and J. Manz, Mol. Phys., 29, 347 (1975).

[6] G. C. Schatz and A. Kuppermann, J. Chem. Phys., 65, 4668 (1976).

[7] F. Fernández-Alonso and R. N. Zare, Annu. Rev. Phys. Chem., 53, 67 (2002).

[8] M. J. Redmon and R. E. Wyatt, Chem. Phys. Lett., 63, 209 (1979).

[9] D. E. Manolopoulos, K. Stark, H.-J. Werner, D. W. Arnold, S. E. Bradforth, and D. M. Neumark, Science, 262, 1852 (1993).

[10] K. Stark and H.-J. Werner, J. Chem. Phys., 104, 6515 (1996).

[11] D. C. Clary, J. Chem. Phys., 95, 7298 (1991).

[12] S. K. Pogrebnya, J. Palma, D. C. Clary, and J. Echave, Phys. Chem. Chem. Phys., 2, 693 (2000). 
[13] D. H. Zhang and J. Z. H. Zhang, J. Chem. Phys., 101, 1146 (1994).

[14] S. Bhattacharya, A. N. Panda, and H.-D. Meyer, J. Chem. Phys., 135, 194302 (2011).

[15] M. T. Cvitaš and S. C. Althorpe, J. Chem. Phys., 139, 064307 (2013).

[16] J. Chen, X. Xu, X. Xu, and D. H. Zhang, J. Chem. Phys., 138, 154301 (2013).

[17] A. Li and H. Guo, J. Phys. Chem. A, 118, 11168 (2014).

[18] D. C. Clary, J. Phys. Chem., 98, 10678 (1994).

[19] G. Nyman and D. C. Clary, J. Chem. Phys., 100, 3556 (1994).

[20] D. C. Clary and J. Palma, J. Chem. Phys., 106, 575 (1997).

[21] J. Palma and D. C. Clary, J. Chem. Phys., 112, 1859 (2000).

[22] R. Welsch and U. Manthe, J. Chem. Phys., 142, 064309 (2015).

[23] Y. V. Suleimanov, R. Collepardo-Guevara, and D. E. Manolopoulos, J. Chem. Phys., 134, 044131 (2011).

[24] B. Kerkeni and D. C. Clary, J. Chem. Phys., 120, 2308 (2004).

[25] B. Kerkeni and D. C. Clary, Phys. Chem. Chem. Phys., 8, 917 (2006).

[26] S. C. Althorpe and D. C. Clary, Annu. Rev. Phys. Chem., 54, 493 (2003).

[27] H. Eyring, J. Chem. Phys., 3, 107 (1935). 
[28] D. G. Truhlar, B. C. Garrett, and S. J. Klippenstein, J. Phys. Chem., 100, 12771 (1996).

[29] W. H. Miller, J. Chem. Phys., 62, 1899 (1975).

[30] W. H. Miller, Faraday Discuss. Chem. Soc., 62, 40 (1977).

[31] W. H. Miller, R. Hernandez, N. C. Handy, D. Jayatilaka, and A. Willetts, Chem. Phys. Lett., 172, 62 (1990).

[32] M. J. Cohen, A. Willetts, and N. C. Handy, J. Chem. Phys., 99, 5885 (1993).

[33] J. R. Barker, T. L. Nguyen, and J. F. Stanton, J. Phys. Chem. A, 116, 6408 (2012).

[34] T. L. Nguyen, J. F. Stanton, and J. R. Barker, J. Phys. Chem. A, 115, 5118 (2011).

[35] S. M. Greene, X. Shan, and D. C. Clary, J. Phys. Chem. A, 119, 12015 (2015).

[36] E. B. Stechel, R. B. Walker, and J. C. Light, J. Chem. Phys., 69, 3518 (1978).

[37] S. T. Banks and D. C. Clary, J. Chem. Phys., 130, 024106 (2009).

[38] S. T. Banks, C. S. Tautermann, S. M. Remmert, and D. C. Clary, J. Chem. Phys., 131, 044111 (2009).

[39] S. M. Remmert, S. T. Banks, and D. C. Clary, J. Phys. Chem. A, 113, 4255 (2009). 
[40] H. F. von Horsten, S. T. Banks, and D. C. Clary, J. Chem. Phys., 135, 094311 (2011).

[41] J. T. Muckerman, Chem. Phys. Lett., 173, 200 (1990).

[42] J. C. Light, I. P. Hamilton, and J. V. Lill, J. Chem. Phys., 82, 1400 (1985).

[43] J. Echave and D. C. Clary, Chem. Phys. Lett., 190, 225 (1992).

[44] J. C. Light, in D. C. Clary, ed., The Theory of Chemical Reaction Dynamics, 215-234, D. Reidel Publishing Company, 1986.

[45] R. B. Walker and E. F. Hayes, in D. C. Clary, ed., The Theory of Chemical Reaction Dynamics, volume 170 of NATO ASI Series, 105-133, Springer Netherlands, 1986.

[46] J. Römelt, in D. C. Clary, ed., The Theory of Chemical Reaction Dynamics, 77-104, D. Reidel Publishing Company, 1986.

[47] S. T. Banks and D. C. Clary, Phys. Chem. Chem. Phys., 9, 933 (2007).

[48] A. N. Brooks and D. C. Clary, J. Chem. Phys., 92, 4178 (1990).

[49] D. C. Clary, Phys. Chem. Chem. Phys., 1, 1173 (1999).

[50] J. M. Bowman, Theor. Chem. Acc., 108, 125 (2002).

[51] J. M. Bowman, Reaction and Molecular Dynamics, Springer: New York, 2000.

[52] B. Kerkeni and D. C. Clary, J. Chem. Phys., 123, 64305 (2005). 
[53] B. Kerkeni and D. C. Clary, Mol. Phys., 103, 1745 (2005).

[54] B. Kerkeni and D. C. Clary, Chem. Phys. Lett., 438, 1 (2007).

[55] X. Shan and D. C. Clary, J. Phys. Chem. A, 118, 10134 (2014).

[56] J. M. Bowman, B. J. Braams, S. Carter, C. Chen, G. Czakó, B. Fu, X. Huang, E. Kamarchik, A. R. Sharma, B. C. Shepler, Y. Wang, and Z. Xie, J. Phys. Chem. Lett., 1, 1866 (2010).

[57] J. M. Bowman, G. Czako, and B. Fu, Phys. Chem. Chem. Phys., 13, 8094 (2011).

[58] W. Koch and D. H. Zhang, J. Chem. Phys., 141, 021101 (2014).

[59] R. Conte, P. L. Houston, and J. M. Bowman, J. Chem. Phys., 140, 151101 (2014).

[60] J. M. Bowman, in I. Prigogine and S. A. Rice, eds., Advances in Chemical Physics, volume 61, 115-167, John Wiley \& Sons, Inc., Hoboken, 1985.

[61] J. M. Bowman, J. Phys. Chem., 95, 4960 (1991).

[62] D. C. Clary, Science, 279, 1879 (1998).

[63] D. C. Clary, Science, 789 (2008).

[64] H. Szichman, M. Baer, and H. Nakamura, J. Chem. Phys., 107, 3521 (1997).

[65] X. Shan and D. C. Clary, Phys. Chem. Chem. Phys., 15, 1222 (2013). 
[66] S. M. Remmert, S. T. Banks, J. N. Harvey, A. J. Orr-Ewing, and D. C. Clary, J. Chem. Phys., 134, 204311 (2011).

[67] X. Shan, S. M. Remmert, D. C. Clary, B. Zhang, and K. Liu, Chem. Phys. Lett., 587, 88 (2013).

[68] X. Shan and D. C. Clary, Phys. Chem. Chem. Phys., 15, 18530 (2013).

[69] W. H. Miller, N. C. Handy, and J. E. Adams, J. Chem. Phys., 72, 99 (1980).

[70] J. Carrington T. and W. H. Miller, J. Chem. Phys., 81, 3942.

[71] H.-J. Werner, et al., MOLPRO, a package of ab initio programs, version 2010.2 .

[72] Gaussian 09 Revision E.01, Gaussian, Inc., Wallingford (2009).

[73] J. F. Stanton, et al., CFOUR, Coupled-Cluster techniques for Computational Chemistry, a quantum-chemical program package (2009).

[74] T. H. Dunning, J. Chem. Phys., 90, 1007 (1989).

[75] T. B. Adler, G. Knizia, and H.-J. Werner, J. Chem. Phys., 127, 221106 (2007).

[76] G. Knizia, T. B. Adler, and H.-J. Werner, J. Chem. Phys., 130, 054104 (2009).

[77] K. A. Peterson, T. B. Adler, and H.-J. Werner, J. Chem. Phys., 128, 084102 (2008).

[78] J. Hranisavljevic and J. V. Michael, J. Phys. Chem. A, 102, 7668 (1998).

[79] Y. Hidaka, T. Nakamura, H. Kawano, and T. Koike, Int. J. Chem. Kinet., 25, 983 (1993).

[80] N. L. Arthur and T. N. Bell, Rev. Chem. Intermed., 2, 37 (1978).

[81] D. K. Maity, W. T. Duncan, and T. N. Truong, J. Phys. Chem. A, 103, 2152 (1999).

[82] C. L. Kibby and R. E. Weston, Jr., J. Chem. Phys., 49, 4825 (1968). 
[83] B. Kerkeni and D. C. Clary, J. Phys. Chem. A, 107, 10851 (2003).

[84] J. Espinosa-García and J. C. Corchado, J. Chem. Phys., 105, 3517 (1996).

[85] Y. Liu, Z. Liu, G. Lv, L. Jiang, and J. Sun, Chem. Phys. Lett., 423, 157 (2006).

[86] C. Rangel, J. Espinosa-García, and J. C. Corchado, J. Phys. Chem. A, 109, 8071 (2005).

[87] L. Zhang, M.-D. Chen, M.-L. Wang, and K.-L. Han, J. Chem. Phys., 112, $3710(2000)$.

[88] E. Garcia, C. Sanchez, A. Saracibar, and A. Laganà, J. Phys. Chem. A, 108, 8572 (2004).

[89] G. Nyman, J. Zhou, B. Zhang, and K. Liu, Isr. J. Chem., 47, 1 (2007).

[90] S. Skokov and J. M. Bowman, J. Chem. Phys., 113, 4495 (2000).

[91] Z. H. Kim, H. A. Bechtel, and R. N. Zare, J. Am. Chem. Soc., 123, 12714 (2001).

[92] H. A. Bechtel, Z. H. Kim, C. J. P., and R. N. Zare, J. Chem. Phys., 122, 084303 (2005).

[93] J. Zhou, B. Zhang, J. J. Lin, and K. Liu, Mol. Phys., 103, 1757 (2005).

[94] S. Yoon, R. J. Holiday, and F. F. Crim, J. Chem. Phys., 119, 4755 (2003).

[95] B. Zhang and K. Liu, J. Chem. Phys., 122, 101102 (2005).

[96] K. L. Schuchardt, B. T. Didier, T. Elsethagen, L. Sun, V. Gurumoorthi, J. Chase, J. Li, and T. L. Windus, J. Chem. Inf. Model., 47, 1045 (2007).

[97] A. Halkier, T. Helgaker, P. Jorgensen, W. Klopper, H. Koch, J. Olsen, and A. K. Wilson, Chem. Phys. Lett., 286, 243 (1998).

[98] R. M. Marshall, H. Purnell, and P. W. Satchell, J. Chem. Soc., Faraday Trans. 1, 80, 2395 (1984).

[99] R. M. Marshall, H. Purnell, and A. Sheppard, J. Chem. Soc., Faraday Trans. 2, 82, 929 (1986).

[100] A. Galano, J. R. Alvarez-Idaboy, and A. Vivier-Bunge, Theor. Chem. Account, 597-606 (2007). 
[101] P. A. Arnold and B. K. Carpenter, Chem. Phys. Lett., 328, 90 (2000).

[102] N. Cohen, Int. J. Chem. Kinet., 23, 683 (1991).

[103] I. M. Mills, in C. W. Mathews and K. N. Rao, eds., Molecular Spectroscopy: Modern Research, chapter 3.2, 115-140, Academic Press, New York, 1972.

[104] R. Hernandez and W. H. Miller, Chem. Phys. Lett., 214, 129 (1993).

[105] J. E. Wollrab, Rotational Spectra and Molecular Structure, Physical Chemistry: a Series of Monographs, Academic Press, Inc., New York, 1967.

[106] M. J. Cohen, N. C. Handy, R. Hernandez, and W. H. Miller, Chem. Phys. Lett., 192, 407 (1992).

[107] D. J. Griffiths, Introduction to Quantum Mechanics, Pearson Education, Inc., Upper Saddle River, 2005, 2 edition.

[108] A. F. Wagner, J. Phys. Chem. A, 117, 13089 (2013).

[109] Z. Ahmed, Phys. Lett. A, 157, 1 (1991).

[110] D. G. Truhlar, A. D. Isaacson, and B. C. Garrett, in M. Baer, ed., Theory of Chemical Reaction Dynamics, volume IV, chapter 2, 65-137, CRC Press, Boca Raton, 1985.

[111] W. H. Miller, Faraday Discuss., 110, 1 (1998).

[112] T. L. Nguyen and J. R. Barker, J. Phys. Chem. A, 114, 3718 (2010).

[113] J. R. Barker, Int. J. Chem. Kinet., 33, 232 (2001).

[114] J. R. Barker, N. F. Ortiz, J. M. Preses, L. L. Lohr, A. Maranzana, P. J. Stimac, T. L. Nguyen, and T. J. D. Kumar, Multiwell-2014.1 software (2014), http://aoss.engin.umich.edu/multiwell/.

[115] S. M. Greene, X. Shan, and D. C. Clary, J. Chem. Phys., 144, 084113 (2016).

[116] L. F. Richardson and J. A. Gaunt, Phil. Trans. R. Soc. Lond. A, 226, 299 (1927).

[117] C. Ridders, Adv. Eng. Software, 4, 75 (1982).

[118] W. H. Press, S. A. Teukolsky, W. T. Vetterling, and B. P. Flannery, Numerical Recipes in C: The Art of Scientific Computing, Cambridge University Press, Cambridge, 1992, 2 edition. 
[119] S. M. Greene, X. Shan, and D. C. Clary, J. Chem. Phys., 144, 244116 (2016), in press.

[120] W. Schneider and W. Thiel, Chem. Phys. Lett., 157, 367 (1989).

[121] V. Barone, J. Chem. Phys., 122, 14108 (2005).

[122] W. H. Green, D. Jayatilaka, A. Willetts, R. D. Amos, and N. C. Handy, J. Chem. Phys., 93, 4965 (1990).

[123] A. Hoy, I. Mills, and G. Strey, Mol. Phys., 24, 1265 (1972).

[124] R. Khairutdinov, K. I. Zamaraev, and V. P. Zhadanov, in R. G. Compton, ed., Electron Tunneling in Chemistry, volume 30 of Comprehensive Chemical Kinetics, 69-110, Elsevier, Amsterdam, 1989.

[125] R. Welsch and U. Manthe, J. Chem. Phys., 137, 244106 (2012).

[126] Q. Meng, J. Chen, and D. H. Zhang, J. Chem. Phys., 143, 101102 (2015).

[127] M. G. Bryukov, I. R. Slagle, and V. D. Knyazev, J. Phys. Chem. A, 105, 6900 (2001).

[128] J. Lede and J. Villermaux, Can. J. Chem., 56, 392 (1978).

[129] B. Kerkeni and D. C. Clary, J. Chem. Phys., 121, 6809 (2004).

[130] C. S. Tautermann and D. C. Clary, Phys. Chem. Chem. Phys., 8, 1437 (2006).

[131] D. C. Clary and R. K. Nesbet, J. Chem. Phys., 71, 1101 (1979). 